\title{
MINLO: multi-scale improved NLO
}

\author{
Keith Hamilton, ${ }^{a, 1}$ Paolo Nason ${ }^{a, b}$ and Giulia Zanderighi ${ }^{c}$ \\ ${ }^{a}$ Theory Division, CERN, \\ CH-1211, Geneva 23, Switzerland \\ ${ }^{b}$ INFN — sezione di Milano Bicocca, \\ Milan, Italy \\ ${ }^{c}$ Rudolf Peierls Centre for Theoretical Physics, \\ 1 Keble Road, University of Oxford, U.K. \\ E-mail: keith.hamilton@cern.ch, paolo.nason@mib.infn.it, \\ g.zanderighi1@physics.ox.ac.uk
}

ABSTRACT: In the present work we consider the assignment of the factorization and renormalization scales in hadron collider processes with associated jet production, at next-toleading order (NLO) in perturbation theory. We propose a simple, definite prescription to this end, including Sudakov form factors to consistently account for the distinct kinematic scales occuring in such collisions. The scheme yields results that are accurate at NLO and, for a large class of observables, it resums to all orders the large logarithms that arise from kinematic configurations involving disparate scales. In practical terms the method is most simply understood as an NLO extension of the matrix element reweighting procedure employed in tree level matrix element-parton shower merging algorithms. By way of a proof-of-concept, we apply the method to Higgs and Z boson production in association with up to two jets.

KeYwords: QCD Phenomenology, NLO Computations

ARXIV EPRINT: 1206.3572

\footnotetext{
${ }^{1}$ On leave from University College London.
} 


\section{Contents}

1 Introduction 1

2 Summary of the CKKW formalism 4

3 Formulation of the method $\quad 6$

4 Interplay between scale choices and Sudakov form factors 4

5 Phenomenology 10

$\begin{array}{lll}5.1 & \text { Preliminary considerations } & 11\end{array}$

$\begin{array}{lll}5.2 & \text { Higgs boson production } & 13\end{array}$

5.2.1 Higgs boson production in association with one jet 13

5.2.2 NLO Higgs boson production in association with two jets 17

$\begin{array}{lll}5.3 & Z \text { boson production } & 20\end{array}$

5.3.1 $\boldsymbol{Z}$ boson production in association with one jet 20

5.3.2 $Z$ boson production in association with two jets 21

6 Conclusions $\quad 22$

$\begin{array}{lr}\text { A NLL improved Sudakov } & 25\end{array}$

\section{Introduction}

Perturbative QCD calculations depend on unphysical renormalization and factorization scales. Theoretical uncertainties are usually estimated by varying the scales by a factor of two above and below their central value. In practice, in next-to-leading order (NLO) calculations the central scale is often determined a posteriori by requiring either the NLO corrections to be small, or the scale sensitivity to be minimised. These stability criteria are usually motivated on the basis that 'bad' scale choices will give rise to large logarithms of the ratio of the renormalization and/or factorization scale with respect to scales characteristic of the process of interest and, hence, to sizable corrections. These large corrections will induce in turn a stronger scale dependence in the cross sections.

While it is certainly the case that unrepresentative scale choices can lead to poor convergence of fixed order predictions, renormalization and factorization scale logarithms are only one possible cause of large higher order contributions. Significant physical contributions may also arise from a number of other sources such as Sudakov effects, large color factors, large $\pi^{2}$ terms, and the opening up of new channels. Moreover, at least 
the dominant components of these corrections take the form of double logarithmic contributions and hence cannot be absorbed by any judicious choice of renormalization and factorization scales.

As an illustrative example consider the familiar case of vector boson production in hadronic collisions. For fully inclusive observables there is no room for ambiguity in the choice of factorization scale, which is naturally set equal to the mass of the vector boson, since this scale limits the QCD radiation that accompanies the production process. On the other hand, turning to the slightly more subtle case of vector boson production in association with a jet, we are faced with two scales: the vector boson mass and the jet transverse momentum. It is well known that this cross section carries (Sudakov) double logarithms of the ratio of these two scales and that such terms are large in the low transverse momentum region. By choosing the renormalization and factorization scales on the basis that radiative corrections be small, one is led to compensate such genuine physical effects with unphysical scale logarithms. Even if one takes the extreme view that the renormalization and factorization scales are arbitrary parameters that one is free 'to tune' in making predictions, it is difficult to see how the associated uncertainty could be considered reliable or unbiased in the presence of theoretically spurious compensation mechanisms.

In short, the stability criterion is potentially misleading, since it attributes all large NLO corrections to these scale logarithms. Both out of theoretical correctness and pragmatism we are therefore motivated to look for an unbiased method to choose the central scales, based on the kinematics and dynamics of the process under study, rather than an a posteriori observation of stability. ${ }^{1}$

In the context of leading order matrix element-parton shower merging algorithms [4-9], an unbiased method for assigning the factorization and renormalization scales is essential and put to good effect. In practice, the kinematic configuration of the process is associated with the most probable branching history by an exclusive jet clustering algorithm. The transverse momentum at each branching defines the renormalization scale for the corresponding factor of $\alpha_{\mathrm{S}}$ at the vertex, while the factorization scale is associated with the matrix element - parton shower merging scale. Furthermore, a recipe is given for including Sudakov form factors, accounting for the large double logarithms that arise when the clustered event contains well separated scales. The net effect of the scale assignment and Sudakov factors is to incorporate, consistently, all large logarithms, i.e. renormalization, factorization and Sudakov logarithms, associated with rendering the event exclusive with respect to radiation above the matrix element-parton shower merging scale - the scale beneath which the parton shower is used to populate the remaining phase space. ${ }^{2}$

It therefore seems appropriate to adapt the calculation of NLO cross sections such that the Born term is evaluated with the scales and Sudakov form factors prescribed by the CKKW method $[4,7,8]$. In the present work we pursue this possibility and construct such a procedure in accordance with the following generic requirements:

\footnotetext{
${ }^{1}$ We stress that the aim of the present work is not to eliminate the scale dependence using some appropriate recipe for its choice [1-3], but rather to find a physically motivated central value, around which we perform scale variation studies in the usual way.

${ }^{2}$ This is not the case for small $x$ or threshold logarithms, that we are not considering in this context.
} 
- the full result has formal NLO accuracy, therefore the scale variation around the central values is formally of next-to-next-to-leading (NNLO) order;

- the accuracy and the smooth behaviour near the Sudakov regions is comparable to that of the corresponding tree-level calculation in the adopted CKKW scheme;

- the procedure is simple and easily implemented for any NLO parton level generator, requiring only minor work on top of the NLO calculation available.

The procedure we propose is based upon two simple observations. The first one concerns the choice of the renormalization scale $\mu_{R}$. To this end let us note that NLO cross sections have the formal structure

$$
\frac{\mathrm{d} \sigma}{\mathrm{d} \Phi}=\alpha_{\mathrm{S}}^{N}\left(\mu_{\mathrm{R}}\right) B+\alpha_{\mathrm{S}}^{N+1}\left(\mu_{\mathrm{R}}\right)\left[V+N b_{0} \log \frac{\mu_{\mathrm{R}}^{2}}{Q^{2}} B\right]+\alpha_{\mathrm{S}}^{N+1}\left(\mu_{\mathrm{R}}\right) R,
$$

where $B$ denotes the Born term and $R$ the real corrections to it. The virtual corrections are shown in parenthesis, their explicit renormalization scale dependence being proportional to the Born term, where $b_{0}$ is the one loop beta function coefficient

$$
b_{0}=\frac{33-2 n_{\mathrm{f}}}{12 \pi},
$$

and $Q$ is a momentum scale representative of the leading order kinematics. The explicit $\mu_{\mathrm{R}}$ dependence of the virtual corrections is such that the variation of eq. (1.1) with respect to changing the renormalization scale is of order $\alpha_{\mathrm{S}}^{N+2}$; terms of order $\alpha_{S}^{N+1}$ induced by varying $\mu_{\mathrm{R}}$ in the Born and virtual contributions cancel exactly due to the renormalization group equation.

From here it is clear that should we choose to evaluate the $N$ coupling constants in the Born term at different scales $\left\{\mu_{i}\right\}$, as in the matrix-element-parton shower merging algorithms, in order for NLO scale compensation to take place eq. (1.1) must generalise to

$$
\frac{\mathrm{d} \sigma}{\mathrm{d} \Phi}=\prod_{i=1}^{N} \alpha_{\mathrm{S}}\left(\mu_{i}\right) B+\alpha_{\mathrm{S}}^{N+1}\left(\mu_{\mathrm{R}}^{\prime}\right)\left[V+b_{0} \sum_{i=1}^{N} \log \frac{\mu_{i}^{2}}{Q^{2}} B\right]+\alpha_{\mathrm{S}}^{N+1}\left(\mu_{\mathrm{R}}^{\prime \prime}\right) R,
$$

where the scales $\mu_{\mathrm{R}}^{\prime}$ and $\mu_{\mathrm{R}}^{\prime \prime}$ in the virtual and real terms are irrelevant from the point of view of scale compensation: $\alpha_{\mathrm{S}}\left(\mu_{\mathrm{R}}\right)-\alpha_{\mathrm{S}}\left(\mu_{\mathrm{R}}^{\prime}\right) \approx \mathcal{O}\left(\alpha_{\mathrm{S}}^{2}\right)$. In eq. (1.3), scale compensation takes place independently for each of the $\mu_{i}$ that is varied. While it may be a relatively straightforward task to evaluate $N$ coupling constants at $N$ scales for the Born term, virtual corrections in NLO calculations are usually expressed in terms of a single renormalization scale only. However, by simply setting $\mu_{\mathrm{R}}$ in the virtual term to be the geometric mean of the $\mu_{i}$ in eq. (1.3)

$$
\mu_{\mathrm{R}}=\left(\prod_{i=1}^{N} \mu_{i}\right)^{\frac{1}{N}}
$$

and evaluating the $N$ coupling constants in the Born term at scales $\mu_{i}$, we arrive at an expression precisely of the form in eq. (1.3). Equivalently, we can evaluate the virtual term 
at some fixed scale $\mu_{0}$ and explicitly add the following contribution

$$
\alpha_{\mathrm{S}}^{N+1}\left(\mu_{\mathrm{R}}^{\prime}\right) \times b_{0} \sum_{i=1}^{N} \log \frac{\mu_{i}^{2}}{\mu_{0}^{2}} B .
$$

The second ingredient that is needed in order to maintain NLO accuracy has to do with the Sudakov form factors that are included in the Born term in the CKKW approach. These form factors, when expanded in powers of $\alpha_{\mathrm{S}}$, lead to terms of order $\alpha_{\mathrm{S}}^{N+1}$, i.e. of the NLO level of accuracy. These terms should be subtracted in order to maintain NLO accuracy.

The choice of scales in the arguments of each power of $\alpha_{\mathrm{S}}$ in the real and the virtual terms, the exact definition of the subtraction term arising from the expansion of the $\mathrm{Su}-$ dakov form factors, and the inclusion of the Sudakov form factors in the real and virtual terms, remain to a large extent arbitrary as far as the NLO accuracy is concerned. We will however further constrain these choices, in such a way that the virtues of the CKKW result at leading order are maintained once radiative corrections are included. We defer the discussion of these and further details to the main body of the article.

The method presented in this paper can be applied in order to improve the prediction for inclusive quantities in any NLO calculation. It is however particularly advantageous in the context of interfacing NLO calculations to parton shower programs [10, 11]. In the POWHEG framework [11, 12], for example, the underlying Born structure of the event is generated with a probability proportional to the NLO inclusive cross section at a given point in the Born phase space. This cross section can be evaluated using the prescription advocated in the present work, leading to a considerable improvement in reliability near the Sudakov regions.

The paper is organized as follows. In section 2 we review briefly the CKKW method for matrix element-parton shower merging. In section 3 we present in detail our prescription. A theoretical discussion regarding the interplay of the scale choices and Sudakov form factors is given in section 4 . In section 5 , as an example, we apply our method to the case of Higgs and $Z$ production in association with one or two jets. Finally, we present our conclusions in section 6. In the appendix we give the exact expression of the Sudakov form factors at next-to-leading logarithmic (NLL) accuracy that we used to obtain the results presented here.

\section{Summary of the CKKW formalism}

We first briefly summarize the standard CKKW procedure $[4,7,8]$. We consider a production process in hadronic collisions. The CKKW formalism requires that we recursively cluster the coloured partons in the event using a $k_{\mathrm{T}}$-clustering algorithm $[13,14]$, in order to reconstruct the most likely branching history. The $k_{\mathrm{T}}$-clustering should be consistent with the flavour structure, i.e. a pair of partons can only be clustered if it can come from a single parton, and the appropriate flavour is assigned to the parton arising from the merging. At each of the vertices $i(i=1, \ldots, n)$ of the branching history, one assigns a nodal scale $q_{i}$, equal to the relative transverse momentum value at which the clustering 
has taken place. In the CKKW formalism one also assigns a resolution scale $Q_{0}$, meaning that the cross section is interpreted as being inclusive for all radiation below $Q_{0}$.

The recursive procedure ends when no further clustering is possible and we refer to the remaining ensemble of particles as the primary system. ${ }^{3}$ We assign it a scale equal to its invariant mass $Q$. The CKKW cross section is obtained by taking the tree-level matrix element, with the strong couplings associated with each node evaluated at the corresponding scale. The remaining $m=N-n$ powers of the strong coupling ${ }^{4}$ are associated with the primary system, and are evaluated at the scale $Q$. Intermediate lines between nodes $i$ and $j$ in the branching history are furthermore assigned a Sudakov form factor

$$
\frac{\Delta_{f_{i j}}\left(Q_{0}, q_{i}\right)}{\Delta_{f_{i j}}\left(Q_{0}, q_{j}\right)}
$$

where $f_{i j}$ is the flavour of the line joining $i$ and $j$, where $i$ is the node closest to the primary vertex $\left(q_{i}>q_{j}\right)$. External lines have Sudakov form factors equal to $\Delta_{f}\left(Q_{0}, q_{i}\right)$, where $i$ is the node connected to the external line.

The general form of the Sudakov exponent is

$$
\Delta_{f}\left(Q_{0}, Q\right)=\exp \left[-\int_{Q_{0}}^{Q} d q \frac{2 C_{f}}{\pi} \frac{\alpha_{\mathrm{S}}(q)}{q}\left(\log \frac{Q}{q}-B_{f}\right)\right], \quad f=q, g,
$$

where $C_{g}=C_{A}, B_{g}=\pi b_{0} / C_{A}$ or $C_{q}=C_{F}, B_{q}=3 / 4$ for gluon or quark lines respectively. Using the leading logarithmic expression for $\alpha_{\mathrm{S}}$, we can compute the Sudakov form factor analytically. We obtain

$$
\Delta_{f}\left(Q_{0}, Q\right)=\exp \left[-\frac{C_{f}}{\pi b_{0}}\left\{\log \frac{\log \frac{Q^{2}}{\Lambda^{2}}}{\log \frac{Q_{0}^{2}}{\Lambda^{2}}}\left(\frac{1}{2} \log \frac{Q^{2}}{\Lambda^{2}}-B_{f}\right)-\frac{1}{2} \log \frac{Q^{2}}{Q_{0}^{2}}\right\}\right] .
$$

A more detailed analysis, adequate for NLL accuracy, is presented in appendix A.

Expanding eq. (2.3) in powers of $\alpha_{\mathrm{S}}$ we get

$$
\begin{aligned}
\Delta_{f}\left(Q_{0}, Q\right) & =1+\Delta_{f}^{(1)}\left(Q_{0}, Q\right)+\mathcal{O}\left(\alpha_{\mathrm{S}}^{2}\right), \\
\Delta_{f}^{(1)}\left(Q_{0}, Q\right) & =-\frac{C_{f}}{\pi} \alpha_{\mathrm{S}}\left[\frac{1}{4} \log ^{2} \frac{Q^{2}}{Q_{0}^{2}}-\log \frac{Q^{2}}{Q_{0}^{2}} B_{f}\right],
\end{aligned}
$$

that represents the effective NLO correction that is already included in the Born term when we use the CKKW prescription, and will eventually be subtracted in our method.

Finally, we note that in the CKKW algorithm the factorization scale in the parton density functions is set to $Q_{0}$, the matrix element-parton shower merging scale. Each event from the tree-level matrix element generator, when reweighted to include these Sudakov form factor and scale settings, is then passed to a parton shower simulation, constrained

\footnotetext{
${ }^{3}$ In processes like $W+$ jets production, the clustering typically stops when all jets are clustered away. In the case of jet production, clustering should stop when at least two jets are left.

${ }^{4}$ In the case of Higgs production in gluon fusion, for example, there will be always at least two powers of $\alpha_{\mathrm{S}}$ associated with the primary system.
} 
in such a way that no further radiation is generated at scales above $Q_{0}$. Hence, the distribution of radiation resolved at scales above $Q_{0}$ is governed by exact tree-level matrix elements with the remaining phase space filled by the parton shower.

In the CKKW scheme, inclusive configurations with the maximum number of partons in the matrix elements are treated differently [8]. In this case, the scale $Q_{0}$ is taken equal to the lowest merging scale. Hence, when interfacing the reweighted tree level events to the parton shower, all higher jet multiplicities, for which no tree-level matrix element is available, will be consistently generated by the shower.

In the context of our approach, the natural choice of $Q_{0}$ is the same one adopted in the CKKW scheme for the highest multiplicity sample. More specifically, in our case $Q_{0}$ should be set equal to the scale of the first merging for Born level kinematics (i.e. the Born and the virtual), and the scale of the second merging for the real kinematics. This is easily understood with the following example. When we consider $Z+$ jet production, with the jet transverse momentum equal to $p_{T}$, we clearly imply that the jet we are considering is the hardest one and thus that its $p_{T}$ limits the scale of all other jets. In the case of the real emission in an NLO calculation, the lowest merging scale corresponds to integrating over further radiation inclusively, hence, its merging scale (the first one in this case) is skipped.

\section{Formulation of the method}

We now formulate our complete prescription, MINLO for Multi-scale improved NLO, including the choice of scales appearing in the coupling constants associated with the NLO corrections, the inclusion of the Sudakov form factors in the virtual and real contributions, and how to perform the subtraction of the term in eq. (2.5). We recall that $Q$ is the scale of the primary configuration, $q_{1} \ldots q_{n}$ are the remaining clustering scales in increasing order, and that generally we have $m$ powers of $\alpha_{\mathrm{S}}$ (where $m$ can be zero) associated with the primary process. In the case of the real cross section, there will be also a smallest clustering scale $q_{0}$, corresponding to the first clustering. As discussed above, we will always fix the scale $Q_{0}$ entering eq. (2.1) to $q_{1}$. We then proceed as follows:

i. We perform the $k_{\mathrm{T}}$ clustering of the event, determine the scales $Q, q_{1} \ldots q_{n}$, and eventually $q_{0}$ for the real term, and construct the event skeleton. We cluster only partons that are compatible in flavours, i.e. gluons with gluons, yielding gluon pseudopartons, gluons and quarks, yielding quark pseudopartons with the same flavour, and quarks with antiquarks of opposite flavour, leading to gluon pseudopartons. We set $Q_{0}=q_{1}$.

It may occur that the scale of the primary process $Q$ turns out to be smaller than the last clustering scale. This happens, for example in the production of a massive boson recoiling against a hard jet, with transverse momentum larger than the boson mass. In these cases we will take $Q=q_{n}$. Notice that this choice is not fully motivated by the CKKW approach, which instead deals with naturally ordered radiation. Although this case is interesting on its own, being perhaps related to the giant $K$-factor issues [15], we will not pursue it further in the present work. 
ii. $n$ powers of the coupling constant in the Born, virtual and real contributions will be evaluated at the scales $\mu_{1} \ldots \mu_{n}$, with $\mu_{i}=K_{\mathrm{R}} q_{i}(i=1 \ldots n)$ (the value of $\alpha_{\mathrm{S}}$ to be used in the real and virtual contributions for the $(n+m+1)^{\text {th }}$ power of the coupling constant is specified at point VI). $K_{\mathrm{R}}$ is the renormalization scale factor, equal to 1 for the central value, and typically varied between 0.5 and 2 in order to study scale variation uncertainties. The $m$ strong coupling constants associated with the primary system will be taken equal to $K_{\mathrm{R}} Q$.

iii. The renormalization scale explicitly appearing in the virtual corrections is set to $\mu_{\mathrm{R}}=\left(\left(\mu_{Q}\right)^{m} \times \prod_{i=1}^{n} \mu_{i}\right)^{\frac{1}{m+n}}$, while the factorization scale $\mu_{\mathrm{F}}$, appearing explicitly in the collinear subtraction remnants and in all parton densities functions (pdf's), is assigned the scale $K_{\mathrm{F}} q_{1}$, where $K_{\mathrm{F}}$ is the factorization scale factor.

iv. The Sudakov form factors for all the skeleton lines will be included for the Born, virtual and real contributions. For the latter, as already remarked above, we include the Sudakov form factors corresponding to the branching history obtained after the first clustering. Notice that the external lines that join at the first node have $\Delta\left(Q_{0}, q_{1}\right)=1$, since $Q_{0}=q_{1}$.

v. The subtraction of the NLO contribution present in the CKKW Born term is performed by replacing

$$
B \Rightarrow B \times\left(1-\sum_{i j}\left[\Delta_{f_{i j}}^{(1)}\left(Q_{0}, q_{i}\right)-\Delta_{f_{i j}}^{(1)}\left(Q_{0}, q_{j}\right)\right]-\sum_{l} \Delta_{f_{l}}^{(1)}\left(Q_{0}, q_{k_{l}}\right)\right),
$$

where the first sum extends over all pairs of nodes $i, j$, with $q_{i}>q_{j}$, connected by a line of flavour $f_{i j}$, and the second one runs over all external lines $l$ connected to nodes $k_{l}$ (excluding $k_{l}=1$, which vanishes).

vi. For the value of $\alpha_{\mathrm{S}}$ to be used in the $(n+m+1)^{\text {th }}$ power of $\alpha_{\mathrm{S}}$ appearing in the real and virtual cross section, and also appearing in eq. (3.1), we propose

$$
\alpha_{\mathrm{S}}^{(n+m+1)}=\frac{1}{n+m}\left(\sum_{i=1}^{n} \alpha_{\mathrm{S}}\left(\mu_{i}\right)+m \alpha_{\mathrm{S}}\left(\mu_{Q}\right)\right) .
$$

The logic for this choice is the following. Large QCD corrections can be viewed as being associated with the nodal scales in the branching history, and can thus be viewed as an $\alpha_{\mathrm{S}}$ factor evaluated at the nodal scales times the Born cross section, one for each node. The sum of them will lead to a sum of $\alpha_{\mathrm{S}}$ values taken at each nodal scale. As far as the subtraction term in eq. (3.1) is concerned, here we make the same choice performed in the NLO terms, since the subtraction term is meant to subtract large corrections arising in the NLO terms and already resummed when the full Sudakov form factors are multiplied by the Born term.

Notice that in eq. (3.1) we could have instead used the same value of $\alpha_{\mathrm{S}}$ that appears in the Sudakov form factor, rather than the one given in eq. (3.2). By sticking to 
the present choice, we may be artificially reducing the scale dependence of the whole result. The exploration of this alternative, as well as many other possible variations on the method, will be left to future work. The purpose of the present work is just to present the essential features of the method, and thus we will stick to a definite choice among all possible options.

To further motivate the above prescription, we make the following remarks. First of all, the inclusion of Sudakov form factors and running couplings in the NLO corrections, with essentially the same prescription as in the Born term, guarantees that also when NLO corrections are included, we recover in the Sudakov regions the same smooth behaviour that was present in the Born term alone thanks to the CKKW procedure. A second important remark has to do with the form of the subtraction term arising from eq. (3.1). We notice that this term has precisely the same couplings and Sudakov form factors present in the NLO term. It is thus constructed in such a way as to have an optimal cancellation of the large Sudakov logs arising in the NLO corrections, that are already present in exponentiated form in the Born Sudakov form factor.

\section{Interplay between scale choices and Sudakov form factors}

It is often easy to find conflicting motivations for the choice of scale in an NLO calculation. Consider the example of Higgs plus jet production, assuming that the jet momentum is substantially lower than the Higgs mass. This process is of order $\alpha_{\mathrm{S}}^{3}$ at the Born level and one may be inclined to believe that one out of the three powers of $\alpha_{\mathrm{S}}$, being associated with the radiated jet, should be taken of the order of the jet transverse momentum, while the other two should be of the order of the Higgs mass, and that the factorization scale should be an intermediate scale between the two. On the other hand, if we recall that our cross section describes the hardest jet, and should be viewed as inclusive in all radiation with clustering scale below the $p_{T}$ of the jet, we would reach the conclusion that the factorization and renormalization scales for all powers of $\alpha_{\mathrm{S}}$ should be taken equal to the jet transverse momentum. This is because all gluon propagators and external lines (including the incoming ones) are limited in virtuality by the jet transverse momentum (the internal line by kinematics and the external lines because radiation with merging scale above $p_{T}$ is not allowed).

This apparent conflict illustrates how failing to consider the effect of Sudakov form factors when dealing with the choice of the scales can lead to inconsistent conclusions. First of all, we should recall that Sudakov double logarithms are formally more important than renormalization or factorization scale logarithms, since the latter lead only to single logs. Furthermore, it should also be remembered that some sub-leading terms in the Sudakov logarithms are precisely there to compensate the mismatch between different scales at connected vertices. The purpose of this section is to further elaborate upon these points, and to demonstrate that a scale assignment, in the framework of multi-jet processes, cannot be consistently discussed if Sudakov form factors are not properly included.

Consider the simple example of quantum field theories without infrared divergences, like Yukawa theories or $\Phi^{3}$ in 6 space-time dimensions. In such theories, we may look for 


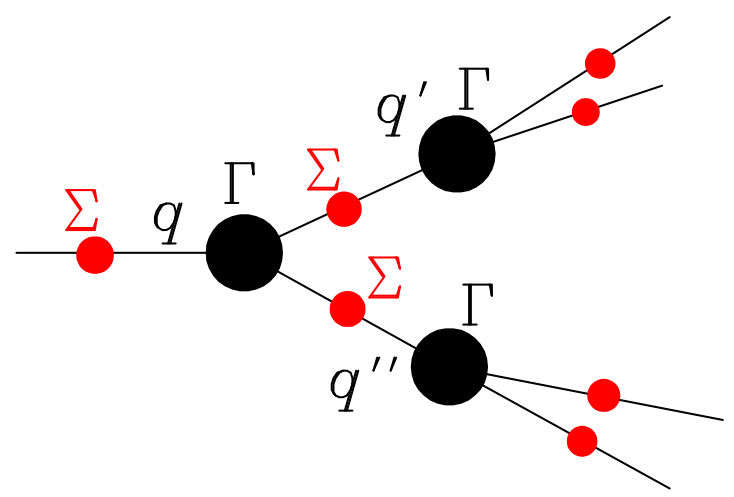

Figure 1. Schematic leading logarithmic corrections to a branching history.

the dominant virtual corrections to a branching process by including leading logarithmic virtual corrections to all vertices and internal lines, as illustrated in figure 1 , where $\Gamma$ represent the one particle irreducible vertex corrections, and $\Sigma$ represent self energies. In these theories (and also in gauge theories in physical gauges), the vertex corrections are infrared finite when at least one external line is off shell, so that they are dominated by the largest virtuality. Thus, in figure 1 , for the leftmost vertex we have

$$
\Gamma\left(q, q^{\prime}, q^{\prime \prime}\right) \approx \Gamma(q, q, q),
$$

and we will write for simplicity

$$
\Gamma(q, q, q)=\Gamma(q)
$$

On the other hand, we have

$$
\Gamma(q)[\Sigma(q)]^{\frac{3}{2}}=\frac{g(q)}{g},
$$

where $g(q)$ is the running coupling at the scale $q$. Thus, if at each vertex with an incoming line having virtuality $q$ we substitute

$$
\Gamma(q)=\frac{g(q)}{g[\Sigma(q)]^{\frac{3}{2}}},
$$

we immediately see that the net effect of the insertion of vertex and self energy corrections is the inclusion of the running coupling constant at the scale of the incoming virtuality for each vertex, and of a factor

$$
\sqrt{\frac{\Sigma\left(q^{\prime}\right)}{\Sigma(q)}}
$$

for each line. This yields in the cross sections, i.e. in the full squared amplitude, a factor

$$
\Delta\left(q^{\prime}, q\right)=\frac{\Sigma\left(q^{\prime}\right)}{\Sigma(q)},
$$

which is the Sudakov form factor.

It is interesting to look in detail to what happens in the case of Higgs plus jet production and how the apparent contradictions arising from naive scale assignments are solved if the 
full Sudakov form factors are included. The couplings and Sudakov factors that multiply the tree level amplitude in the CKKW approach yield the factor

$$
F=\alpha_{\mathrm{S}}^{2}\left(M_{H}\right) \alpha_{\mathrm{S}}\left(p_{T}\right)\left\{\exp \left[-\frac{C_{A}}{\pi b_{0}}\left\{\log \frac{\log \frac{Q^{2}}{\Lambda^{2}}}{\log \frac{Q_{0}^{2}}{\Lambda^{2}}}\left(\frac{1}{2} \log \frac{Q^{2}}{\Lambda^{2}}-\frac{\pi b_{0}}{C_{A}}\right)-\frac{1}{2} \log \frac{Q^{2}}{Q_{0}^{2}}\right\}\right]\right\}^{2} .
$$

Notice that we have only the two powers of the Sudakov form factors, associated with the incoming internal and external lines that join at the Higgs production vertex. The remaining two lines join at the first node, hence their Sudakov form factor is one, since $Q_{0}^{2}=p_{T}^{2}$. Eq. (4.7) in turn leads to

$$
\begin{aligned}
F & =\alpha_{\mathrm{S}}^{2}\left(M_{H}\right) \alpha_{\mathrm{S}}\left(p_{T}\right)\left(\frac{\log \frac{Q^{2}}{\Lambda^{2}}}{\log \frac{Q_{0}^{2}}{\Lambda^{2}}}\right)^{2} \exp \left[-\frac{C_{A}}{\pi b_{0}}\left\{\log \frac{\log \frac{Q^{2}}{\Lambda^{2}}}{\log \frac{Q_{0}^{2}}{\Lambda^{2}}} \log \frac{Q^{2}}{\Lambda^{2}}-\log \frac{Q^{2}}{Q_{0}^{2}}\right\}\right] \\
& \approx \alpha_{\mathrm{S}}^{3}\left(p_{T}\right) \exp \left[-\frac{C_{A}}{\pi b_{0}}\left\{\log \frac{\log \frac{Q^{2}}{\Lambda^{2}}}{\log \frac{Q_{0}^{2}}{\Lambda^{2}}} \log \frac{Q^{2}}{\Lambda^{2}}-\log \frac{Q^{2}}{Q_{0}^{2}}\right\}\right],
\end{aligned}
$$

where we have taken $Q_{0}^{2}=p_{T}^{2}$ and $Q=M_{H}$. Notice that

$$
\exp \left[-\frac{C_{A}}{\pi b_{0}}\left\{\log \frac{\log \frac{Q^{2}}{\Lambda^{2}}}{\log \frac{Q_{0}^{2}}{\Lambda^{2}}} \log \frac{Q^{2}}{\Lambda^{2}}-\log \frac{Q^{2}}{Q_{0}^{2}}\right\}\right] \approx 1-\frac{C_{A}}{\pi} \alpha_{\mathrm{S}} \frac{1}{2} \log ^{2} \frac{Q^{2}}{Q_{0}^{2}}+\mathcal{O}\left(\alpha_{\mathrm{S}}^{2}\right),
$$

i.e. the pure Sudakov double logarithm. Thus, applying the CKKW prescription leads to the conclusion that the scale choice for $\alpha_{\mathrm{S}}$ is $p_{\mathrm{T}}$ for all powers of $\alpha_{\mathrm{S}}$, provided a pure LL Sudakov form factor is included. If one assigns the scale $p_{\mathrm{T}}$ to one power of $\alpha_{\mathrm{S}}$, and $M_{H}$ to the remaining two, then the full NLL Sudakov form factor should be included, that takes care of the scale mismatch.

Thus, the intuitive argument of assigning the same $p_{T}$ scale to all coupling constant is in a sense correct, but one should not forget that double log Sudakov terms are formally more important than scale logarithms.

\section{Phenomenology}

In order to test our prescription, we have implemented it in the POWHEG BOX [16] in a fully generic way, so that it can be applied to any process of interest. In this context, we have performed a variation over the scheme presented in section 3, regarding the first clustering in the real emission contributions. Since the POWHEG BOX already provides a first clustering, corresponding to the mapping of the real emission configuration to its underlying Born structure, we have relied on this mapping rather than performing this clustering explicitly using the $k_{\mathrm{T}}$ algorithm. This procedure is formally equivalent to the one given in section 3 , and it has the advantage of greater simplicity. We have used $R=1$ in our $k_{\mathrm{T}}$ clustering procedure. The Sudakov form factors have been coded both with the expressions of eq. (2.3), and with the full NLL dependence presented in the appendix. It turns out that the two expressions differ very little if the value of $\Lambda$ used in the leading 
order expression is taken equal to $\Lambda_{\overline{\mathrm{MS}}}$. However, we have produced our results using for the Sudakov exponent the full expression given in appendix A.

Our study will focus on two examples: Higgs production via gluon fusion and $Z$ production, both in association with one or two jets. The (infinite top mass limit) Higgs production NLO calculations are taken from refs. [17-21]. They will be referred to as the HJ (for Higgs plus one jet) and HJJ (for Higgs plus two jets) in the present work. The $Z+$ jet cross section ( $\mathrm{ZJ}$ in this paper) is taken from ref. [22]. A $Z+2$ jets POWHEG BOX implementation has appeared in ref. [23]. However, the relevant code is not fully public. We thus implemented a new $Z+2$ jets (ZJJ) POWHEG BOX generator using the automatic MadGraph4 interface developed in ref. [21], taking the virtual corrections from the MCFM package [24-26].

We will refer to the results obtained with the method presented in this work as MINLO (for Multi-scale Improved NLO). All the calculations are performed for the LHC at a centre of mass energy of $7 \mathrm{TeV}$. The Higgs mass is always taken equal to $120 \mathrm{GeV}$. We have used the CTEQ6M parton density functions [27] for Higgs production, MSTW2008NLO [28] for Z production processes, and the $k_{T}$ algorithm for jets, with $R=0.5$, as implemented in Fast Jet [29]. In the Higgs boson production case, the full cross section is reported, with no branching ratios. All results concerning $Z$ production include the branching fraction for $Z \rightarrow e^{+} e^{-}$. A mass window from $60 \mathrm{GeV}$ to $M_{Z}+15 \Gamma_{Z}$ was used for the $Z$ virtuality $\left(\Gamma_{Z}=2.495 \mathrm{GeV}\right)$, while for the Higgs Boson virtuality we have considered the window from $M_{H}-15 \Gamma_{H}$ to $M_{H}+15 \Gamma_{H}\left(\Gamma_{H}=5.75 \cdot 10^{-3} \mathrm{GeV}\right)$.

When showing showered POWHEG results for comparison, these will always be generated interfacing POWHEG with PYTHIA 6.4.25 [30], using the Perugia-0 tune (PYTUNE(320)), with hadronization and underlying event turned off.

We will compare the MINLO results also to standard NLO results obtained with conventional scale choices. In particular, a fixed scale choice, labelled 'FXD' in the figures, will correspond to the scales central values equal to the mass of the heavy boson in all cases. A running scale (labelled 'RUN') will also be considered. It will be taken equal to the jet transverse momentum in both the $H+1$ jet and $Z+1$ jet processes, since this is the scale that one would adopt following the intuitive reasoning of section 4 . In the $H+2$ jets case, the running scale will be taken equal to $\hat{H}_{T}$, defined as

$$
\hat{H}_{T}=\sqrt{M_{H}^{2}+p_{\mathrm{T}}^{H}}+\sum_{i} p_{\mathrm{T}}^{(i)},
$$

where the sum runs over all partons in the event. In the $Z+2$ jets case, the running scale will be taken equal to $\hat{H}_{T} / 2$. The $\hat{H}_{T}$ scale is quite popular in multijet processes, and, in particular, $\hat{H}_{T} / 2$ seems to be the preferred scale for $W$ and $Z$ production in association with jets [31].

\subsection{Preliminary considerations}

Before discussing the goal of our study, it is useful to clarify what we expect from our method by making a couple of consideration regarding the CKKW algorithm when applied to the tree level cross sections. Consider for example, Higgs production plus $n$ partons. 
Because of the unitarity of the shower, in the parton shower approximation of the Higgs plus $n$ partons process, by integrating over the last splitting, one recovers exactly the shower approximation to the cross section for Higgs plus $(n-1)$ partons. One expects something similar to happen for the CKKW formula. ${ }^{5}$ However, in the CKKW case unitarity is not exact, and this feature is only approximate. In the simplest case of a single radiated parton we can easily prove that by integrating out the radiated parton we recover the Born cross section up to corrections of order $\alpha_{\mathrm{S}}$. In the case of more complex configurations, sub-leading logarithms can arise, and a sound conclusion is more difficult to reach.

In order to assess the performance of a MINLO result, we need to compare it to other calculations that give a reasonably good description of the Sudakov region. So, for example, we will compare the MINLO HJ result to the showered, parton level POWHEG result for inclusive Higgs (ggH). If we look, for instance, at the Higgs transverse momentum distribution, the POWHEG ggH result gives a correct description of the Sudakov region and, furthermore, its integral yields the NLO accurate total Higgs production cross section. On the other hand, it describes the tail of the Higgs transverse momentum distribution only with LO accuracy. By contrast, the MINLO result is instead NLO accurate at relatively large transverse momenta and LO accurate for the integral of the whole distribution.

In the case of heavy boson production in association with two jets, like in Higgs plus dijet production, we will compare the MINLO HJJ result with the POWHEG HJ one, enhanced with the MINLO prescription. Here we expect the POWHEG HJ result to give a good description of the Sudakov region associated with the emission of the second parton. Integrating out the second parton emission in distributions that are inclusive in the hardest jet, one achieves NLO accuracy. On the other hand, only LO accuracy is achieved for the production of two widely separated jets. Conversely, the MINLO HJJ calculation has full NLO accuracy for Higgs plus two jets and leads to LO accuracy for Higgs plus one jet distributions.

We remark here that the standard NLO calculations are not integrable over the full phase space. Thus, for example, the HJJ standard NLO result does not yield a finite cross section for Higgs plus one jet distributions, while in the MINLO approach a sensible result is obtained (thanks to the damping of the Sudakov form factors), although only accurate at leading order.

The fact that the MINLO NLO calculation is finite is a remarkable advantage over the usual fixed order calculations, since, for instance, when generation cuts are imposed in order to obtain finite cross sections, one needs to make sure that the cuts are low enough so that final results are not sensitive to them. However, making generation cuts too low renders NLO calculations inefficient, so that usually an appropriate, delicate compromise needs to be found. Another feature that is worth stressing in the MINLO result is the improved stability of the inclusive distributions as the Sudakov regions are reached. This is not only due to the Sudakov suppression factor, but also to the fact that Sudakov logarithms arising at fixed order in the NLO corrections are compensated by the inclusion of the subtraction terms of eq. (3.1), which have exactly the same structure.

\footnotetext{
${ }^{5}$ Notice that, because of the presence of the Sudakov form factors, the CKKW formula is integrable in the full phase space, provided we avoid integrating over the Landau pole of the running coupling constant by, for example, freezing the coupling at a scale just above it.
} 
The observables for which we expect most advantges from the MINLO method are those that can be constructed from the momenta of the pseudo-partons after a $k_{\mathrm{T}}$-clustering procedure carried out until we have $n$ jets, $n$ being the number of radiated partons beyond the primary process at the Born level (e.g. $n=1$ for HJ and ZJ and $n=2$ for HJJ and ZJJ). Strictly speaking it should work for observables built up with the $n$-jet exclusive cross section. This is obtained by applying the $k_{T}$ clustering algorithm, discarding or merging the pseudoparton with the smallest transverse momentum until we are left with exactly $n$ pseudopartons. In practice, it should also work well for quantities built out of the hardest $n$ jets, as defined in the inclusive $k_{\mathrm{T}}$ algorithm with a reasonable (i.e. not too small) choice of the $R$ parameter. We remark, however, that quantities that are sensitive to the radiation in the real event (i.e. to the third parton in HJJ and to the second parton in HJ) the MINLO method has no great advantage over the standard ones. In fact, no Sudakov suppression is included for the radiated parton in the real cross section. On the other hand, the POWHEG method provides specifically these Sudakov form factors, while maintaining NLO accuracy. Therefore, the MINLO method combined with POWHEG yields the fully resummed results for all quantities. We expect that in this framework the POWHEG results improved with the MINLO method will ease the task of merging multijet samples, by providing associated jet cross section that merge more smoothly with those with smaller multiplicity.

It is possible to conceive observables for which the MINLO method includes double logarithms (at the NNLO level and beyond) that are actually not correct [32]. At the end of section 5.2.1 we will consider two such examples.

\subsection{Higgs boson production}

\subsubsection{Higgs boson production in association with one jet}

We begin by considering the MINLO improved HJ calculation. In figure 2 we show the transverse momentum spectrum of the Higgs boson, computed with the POWHEG BOX ggH generator, the HJ-MINLO result, and the HJ result with the two alternative scale choices $\mu_{\mathrm{F}}=\mu_{\mathrm{R}}=p_{\mathrm{T}}^{H}(\mathrm{RUN})$ and $\mu_{\mathrm{F}}=\mu_{\mathrm{R}}=M_{H}$ (FXD). The POWHEG BOX result was obtained with the settings advocated in ref. [33], that is to say with the hdamp parameter set to the Higgs mass divided by 1.2, and all other parameters at their default value. The events were showered at the parton level using PYTHIA 6, with the settings described in the introduction of section 5 .

In the right panel in figure 2, the full scale variation of the HJ results, normalized to the POWHEG BOX result, are presented. We have varied the renormalization and factorization scale independently by a factor of two above and below the central value, discarding the extreme cases of varying them in opposite directions. More precisely, referring to the notation of section 3 , we have chosen $K_{\mathrm{R}}$ and $K_{\mathrm{F}}$ equal to $1 / 2,1$ and 2 , restricted by the condition $1 / 2 \leq K_{\mathrm{R}} / K_{\mathrm{F}} \leq 2$. This leaves seven scales combinations. The upper (lower) curves are obtained by taking the upper (lower) envelope of these seven curves.

Notice the striking difference between the MINLO result and the standard NLO ones. The MINLO result mimics well the POWHEG BOX result down to very low values of transverse momentum. We stress again that this is a consequence of the presence of Sudakov form 

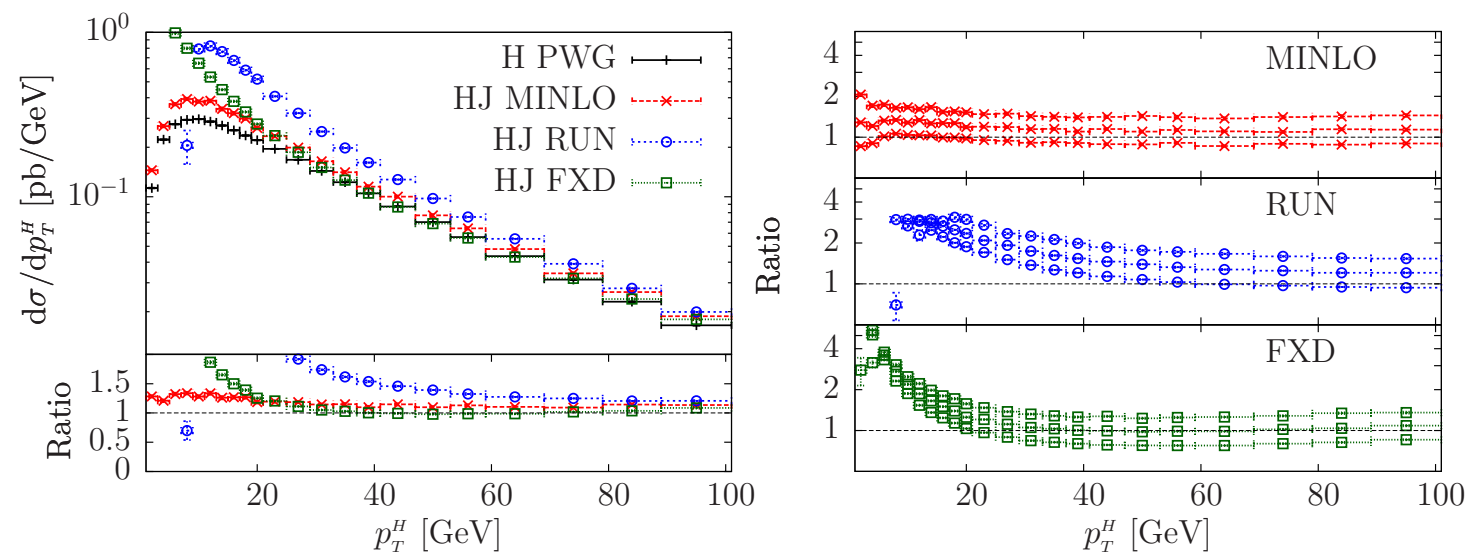

Figure 2. Transverse momentum spectrum of the Higgs boson, computed with the POWHEG BOX ggH generator (H PWG), the HJ-MINLO result (HJ MINLO), the HJ default $\mu_{\mathrm{F}}=\mu_{\mathrm{R}}=p_{\mathrm{T}}^{H}$ (HJ RUN), and HJ with $\mu_{\mathrm{F}}=\mu_{\mathrm{R}}=M_{H}$ (HJ FXD). The right panel shows the ratio of each of the NLO HJ results with respect to the NLO ggH POWHEG simulation with the band either side of the central values indicating the combined renormalization and factorization scale uncertainty. Results are shown for LHC collisions at $7 \mathrm{TeV}$ and a Higgs mass of $120 \mathrm{GeV}$. No cuts are applied.

factors, and also of the inclusion of the subtraction term of eq. (3.1). The standard HJ results do instead diverge at small transverse momentum. Furthermore, they tend to abruptly change sign, due to the growing of the large Sudakov double logarithms arising at the NLO level. Notice also that they begin to depart from the MINLO result even at moderate values of the transverse momenta. By contrast, we observe that the MINLO uncertainty band is fairly compatible with the POWHEG result down to very low values of the transverse momentum.

We notice that the fixed scale result is more compatible with the MINLO result than the running scale one. This may seem surprising, since, as shown in section 4, the MINLO scale choice corresponds to the running scale case. However, the Sudakov suppression of the MINLO result is missing in the running scale result. Using a larger scale at small transverse momenta, as is done in the fixed scale case, compensates to some extent the lack of a Sudakov form factor, yielding a more stable result.

We also remark that the MINLO result yields an increasing scale band at low transverse momenta, i.e. the ratio of the upper to lower band increases at small transverse momenta. This is to be contrasted with the fixed scale case, where the uncertainty band seems to shrink at small $p_{\mathrm{T}}$, giving the illusion of a smaller theoretical uncertainty. The ratio plots in the right panel of figure 2 are shown in logarithmic scale, in order to makes this effect more evident. This observation is easily explained. The NLO correction includes a dominant, negative Sudakov term, carrying two more powers of $\log \left(M_{H} / p_{\mathrm{T}}\right)$ than the Born term. This term causes the NLO correction to change sign at some small value of $p_{\mathrm{T}}$. At the point where the NLO correction becomes zero, its derivative with respect the renormalization scale almost vanishes. In fact, at the point where the NLO correction vanishes, the scale dependence is given schematically by

$$
\sigma=B \alpha_{\mathrm{S}}^{N}\left(\mu_{\mathrm{R}}\right)+N B b_{0} \log \left(\mu_{\mathrm{R}}^{2} / \mu_{0}^{2}\right) \alpha_{\mathrm{S}}^{N+1}\left(\mu_{\mathrm{R}}\right)
$$



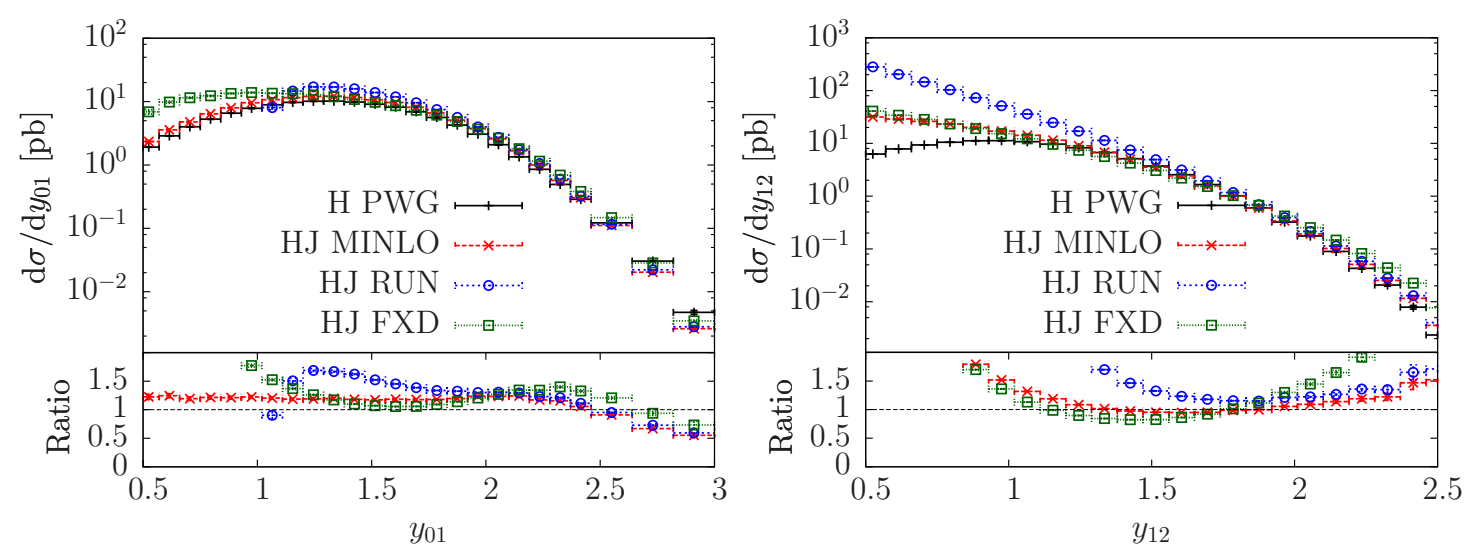

Figure 3. As figure 2 for the $0 \rightarrow 1$ and $1 \rightarrow 2$ differential jet rates in Higgs boson production via gluon fusion.

where $\mu_{0}$ is the scale central value. Its derivative at $\mu_{\mathrm{R}}=\mu_{0}$ is

$$
\mu_{\mathrm{R}}^{2} \frac{d \sigma}{d \mu_{\mathrm{R}}^{2}}=N B \alpha_{\mathrm{S}}^{N}\left(\mu_{\mathrm{R}}\right)\left(-b_{0} \alpha_{\mathrm{S}}\left(\mu_{\mathrm{R}}\right)-b_{1} \alpha_{\mathrm{S}}^{2}\left(\mu_{\mathrm{R}}\right)\right)+N B b_{0} \alpha_{\mathrm{S}}^{N+1}\left(\mu_{\mathrm{R}}\right)=-N B \alpha_{\mathrm{S}}^{N+2}\left(\mu_{\mathrm{R}}\right) b_{1} .
$$

Thus, the scale dependence in this region, being only due to the NLO evolution term of $\alpha_{s}$, is small, and the NLO correction is also small, yielding a full NLO result close to the Born one. Both these conditions may convey a false impression of reliability. ${ }^{6}$ For the MINLO result, on the other hand, this mechanism does not operate, since the large double logarithmic term is removed from the NLO correction, and is included in the Sudakov form factors.

In figure 3 we show the differential jet rates. These are defined as

$$
y_{i i+1}=\log _{10}\left(\frac{q_{i i+1}}{1 \mathrm{GeV}}\right),
$$

where $q_{i i+1}$ is the $k_{\mathrm{T}}$ merging scale for going from a $(i+1)$-jet configuration to a $i$-jet configuration in the $k_{\mathrm{T}}$ clustering procedure. For the $0 \rightarrow 1$ differential jet rate, as for the Higgs transverse momentum distribution, all three methods we are considering should be predictive. We see again that the MINLO prediction is well behaved even below $q_{01} \approx$ $10 \mathrm{GeV}$, while the standard methods fail in this region. The $1 \rightarrow 2$ differential jet rate is a distribution for which we expect little or no improvement from the MINLO method. In fact, it is determined by the distribution of the radiated parton in real events, that forms the second jet. No Sudakov improvement for this emission is provided by the MINLO method. In the POWHEG Higgs implementation, this quantity is determined by the shower stage of the generation, where partons beyond the first one are generated. In a POWHEG simulation of the HJ process, either with the MINLO improvement or with a standard choice of scales, all curves would be in better agreement with the POWHEG ggH result, since in this case a Sudakov form factor for the radiated parton is properly included.

In figure 4 the transverse momentum of the hardest jet is plotted (this distribution is equivalent to $y_{01}$ but shown in a different form and range). This distribution should be

\footnotetext{
${ }^{6}$ See also figure 3 in ref. [34], and the associated discussion.
} 

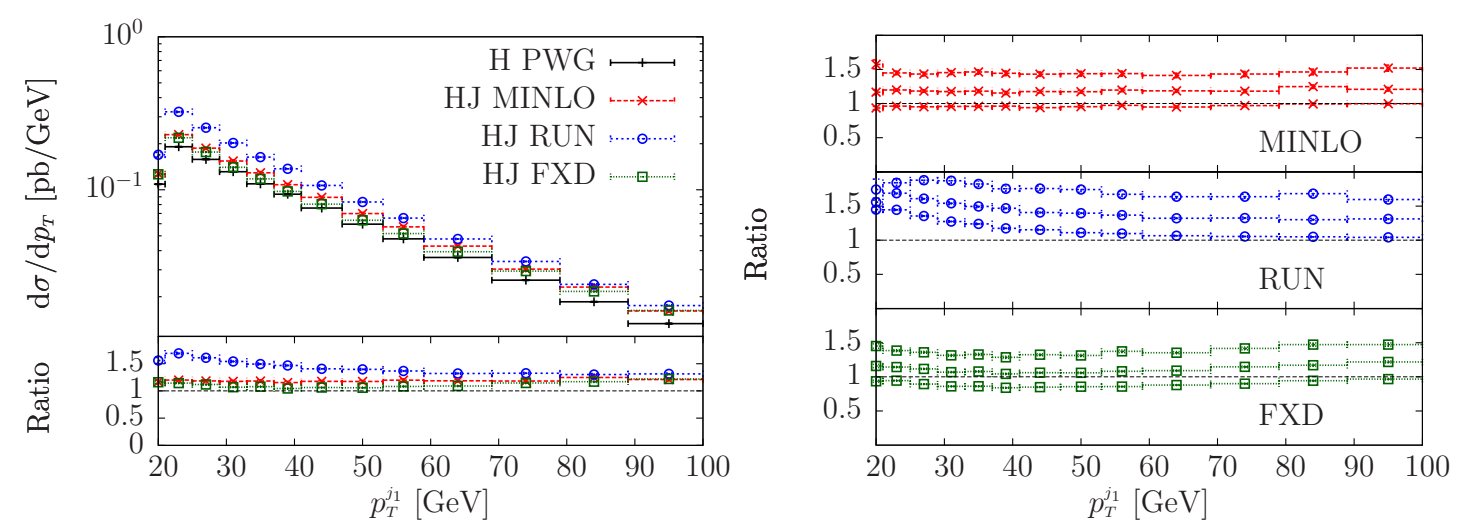

Figure 4. As figure 2 for the transverse momentum spectrum of the leading jet produced in Higgs boson production via gluon fusion.
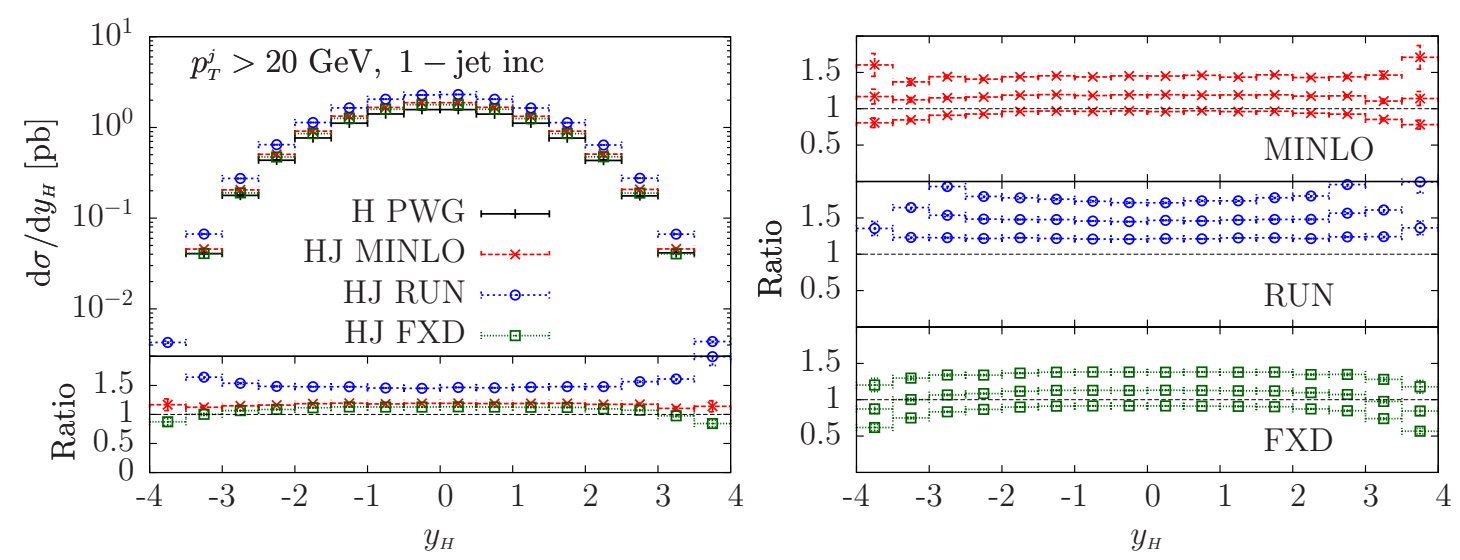

Figure 5. As figure 2 for the rapidity of the Higgs boson in events containing at least one jet with $p_{T}^{j}>20 \mathrm{GeV}$.

similar to the transverse momentum of the Higgs, except for the fact that it is displayed for $p_{T}>20 \mathrm{GeV}$. As the small $p_{\mathrm{T}}$ region is approached, we see indications of an initial unphysical behaviour in the standard methods, especially evident for the running scale case.

In figure 5 we show the rapidity distribution of the Higgs, in events with at least one jet above $20 \mathrm{GeV}$. The normalization of this distribution inherits the results obtained for the jet transverse momentum distribution, since it is mainly affected by the $20 \mathrm{GeV}$ cut. An interesting trend is observed, however, in the large rapidity region, where phase space restrictions become operative and Sudakov effects may become manifest. The MINLO result is more compatible with the POWHEG ggH one, while the default running scale result markedly departs from it.

We consider now two distributions such that the double logarithmic structure introduced with the MINLO procedure is not correct beyond the NLO level [32]. Consider for example the transverse momentum distribution of the hardest jet included in a given rapidity range around the Higgs boson and of the hardest jet in a fixed (central) rapidity region. Observe that in both cases the jet in the considered rapidity range is not necessarily 

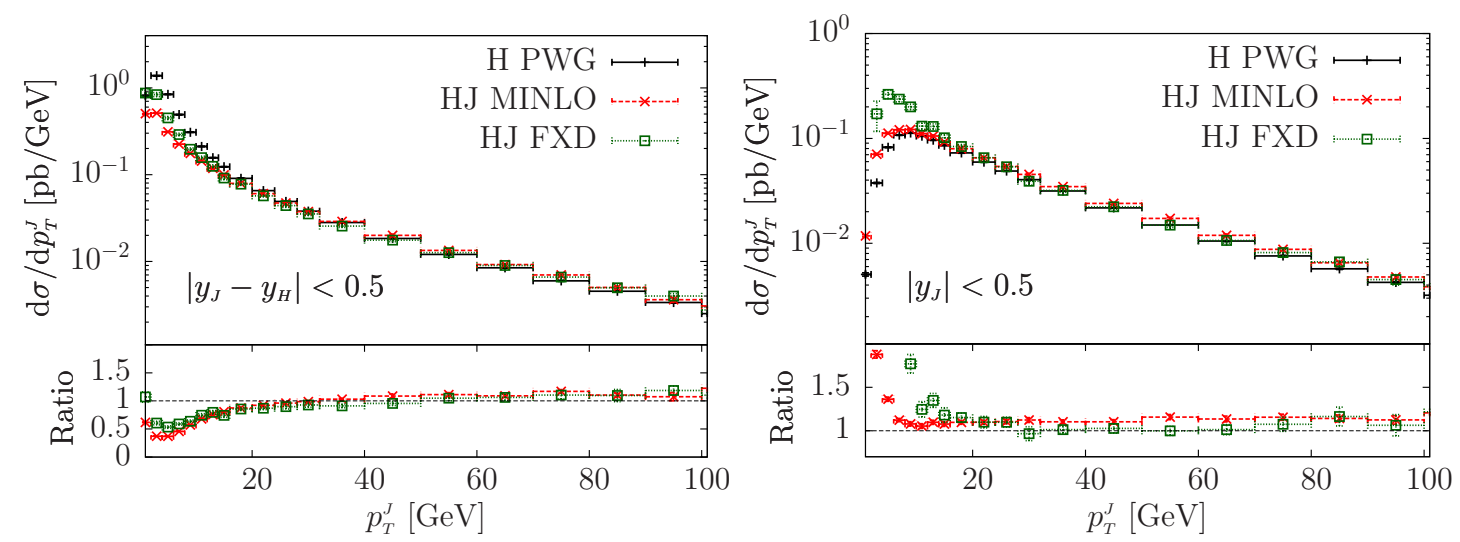

Figure 6. The transverse momentum distribution of the hardest jet with $\left|y_{J}-y_{H}\right|<0.5$ (left plot) and with $\left|y_{J}\right|<0.5$ (right plot).

the hardest one in the whole process, since the hardest jet could be outside that range. Thus, these distributions do not satisfy our requirement mentioned earlier, i.e. they cannot be constructed neither out of the exclusive $k_{\mathrm{T}}$-clustered configuration at the one-jet level, nor out of the inclusive hardest jet distribution. Since we are limiting the collinearity of the emitted jet, the MINLO procedure introduces an excessive double logarithmic Sudakov suppression for these observables. In figure 6 we show predictions for the transverse momentum distribution of the hardest jet with $\left|y_{J}-y_{H}\right|<0.5$ (left plot) and $\left|y_{J}\right|<0.5$ (right plot) from, the showered ggH generator, the MINLO procedure and a standard NLO calculation with a fixed scale. We observe that indeed for the left plot the MINLO prediction seems to fare worse than the standard fixed order NLO computation. On the other hand, relevant differences between the two are only visible for very small $p_{\mathrm{T}}$, already in the region where both distributions depart from the ggH prediction. Conversely, for the right plot the MINLO prediction tracks the ggH one more closely down to smaller values of $p_{\mathrm{T}}$. Even for the left plot, the MINLO result is closer to the ggH one as soon as one increases the rapidity interval. We thus see that even for observables conceived to expose the limitations of the MINLO method, it still performs comparably to standard NLO calculations. Nevertheless, we believe that more extensive experience of the MINLO method is needed in order to fully assess its performance.

\subsubsection{NLO Higgs boson production in association with two jets}

In this section we compare the MINLO HJJ distributions with the standard ones, obtained with two choices of the scale, $\mu_{\mathrm{F}}=\mu_{\mathrm{R}}=M_{H}$, and $\mu_{\mathrm{F}}=\mu_{\mathrm{R}}=\hat{H}_{T}$, with $\hat{H}_{T}$ defined as

$$
\hat{H}_{T}=\sqrt{{M_{H}}^{2}+p_{\mathrm{T}}^{H}}+\sum_{i} p_{\mathrm{T}}^{(i)},
$$

the sum running on all final state partons. These two scale choices will be labelled FXD and RUN in the figures. We begin by comparing in figure 7 the transverse momentum of the Higgs obtained with the POWHEG BOX HJ generator (interfaced to the PYTHIA shower) and the MINLO HJJ generator. The POWHEG BOX HJ generator was modified with the inclusion 

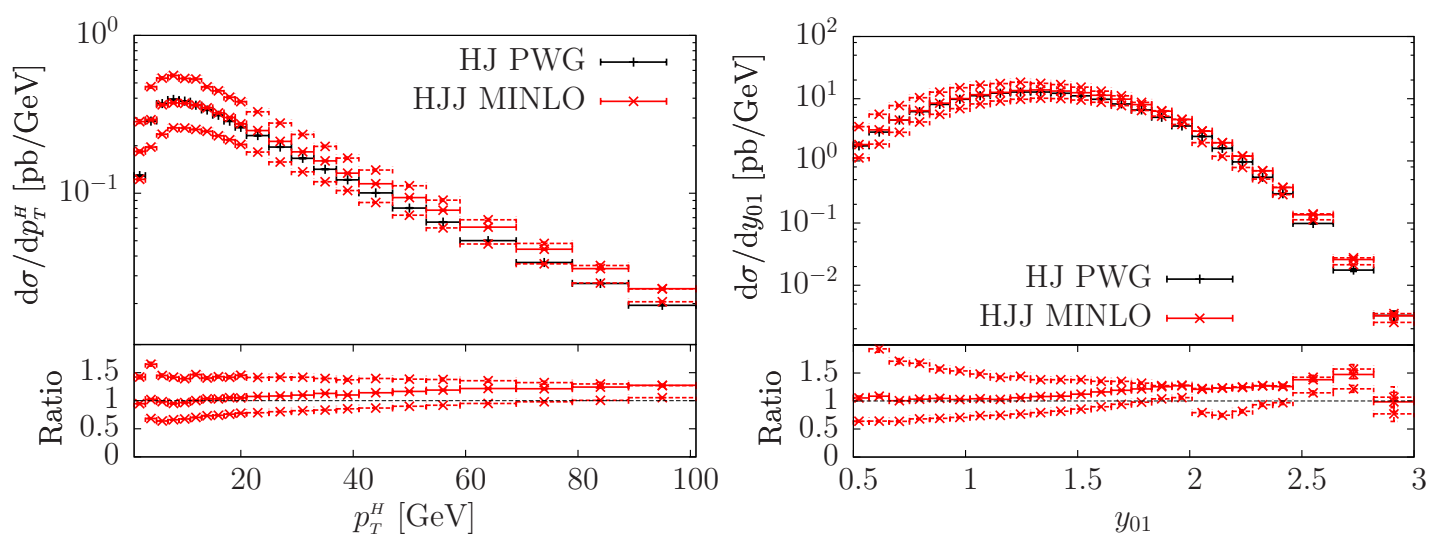

Figure 7. The transverse momentum of the Higgs boson (left) and the differential jet rate $y_{01}$ (right), representing the logarithm of the resolution scale in the $k_{\mathrm{T}}$ jet algorithm [14] for which 1-jet events become resolved as 0-jet ones. Results shown are computed with the POWHEG BOX HJ generator, augmented by the MINLO procedure, and with HJJ-MINLO method. Distributions are shown for LHC collisions at $7 \mathrm{TeV}$ and a Higgs mass of $120 \mathrm{GeV}$. No cuts are applied.

of the MINLO method for the computation of the underlying Born kinematics. No standard NLO Higgs plus two jets prediction is possible for this distribution, since it does not require the presence of at least two jets. Thus, as previously discussed, we expect the MINLO result to give a LO representation of the physical cross section. We can see that, in spite of this the MINLO result is still remarkably close to the POWHEG BOX cross section. The agreement is particularly impressive at very low transverse momentum, where it seems that the MINLO HJJ result gives a description of the total Higgs cross section that is very close to the one given by the HJ POWHEG BOX generator. The latter, when improved with the MINLO prescription, yields a cross section that is accurate at least at LO, according to the discussion given at the beginning of section 5. In the right panel of figure 7 we show the differential jet rate for the zero jet to one jet transition. Here again we see the MINLO prediction closely tracks the result of the HJ POWHEG generator.

In figure 8 the differential jet rate $y_{12}$ is shown. For this distribution the MINLO result and the standard NLO calculations are all predictive, showing reasonable agreement among each other for moderately large merging scales. At small scales, the MINLO result is in better agreement with the POWHEG BOX HJ code and shows a better scale stability. The standard HJJ NLO results, by constrast, display unphysical behaviour under scale variation, especially as far as the $\hat{H}_{T}$ scale choice is concerned.

In figure 9 we show the transverse momentum of the leading jet in events with at least two jets. All NLO calculations, MINLO-improved and those with conventional scale setting, are again predictive for this distribution. Observe that in the case of the running scale prediction $\left(\mu_{\mathrm{R}}=\mu_{\mathrm{F}}=\hat{H}_{T}\right)$ the central value is outside the MINLO error band. Using a central value of $\hat{H}_{T} / 2$ would instead lead to much better agreement between the MINLO and RUN results. Remarkably, it has become common in multijet NLO calculation to prefer $\hat{H}_{T} / 2$ as central scale, because it seems to lead to an improved scale stability. The MINLO result seems also to favour this choice. We also notice that the uncertainty band for the 

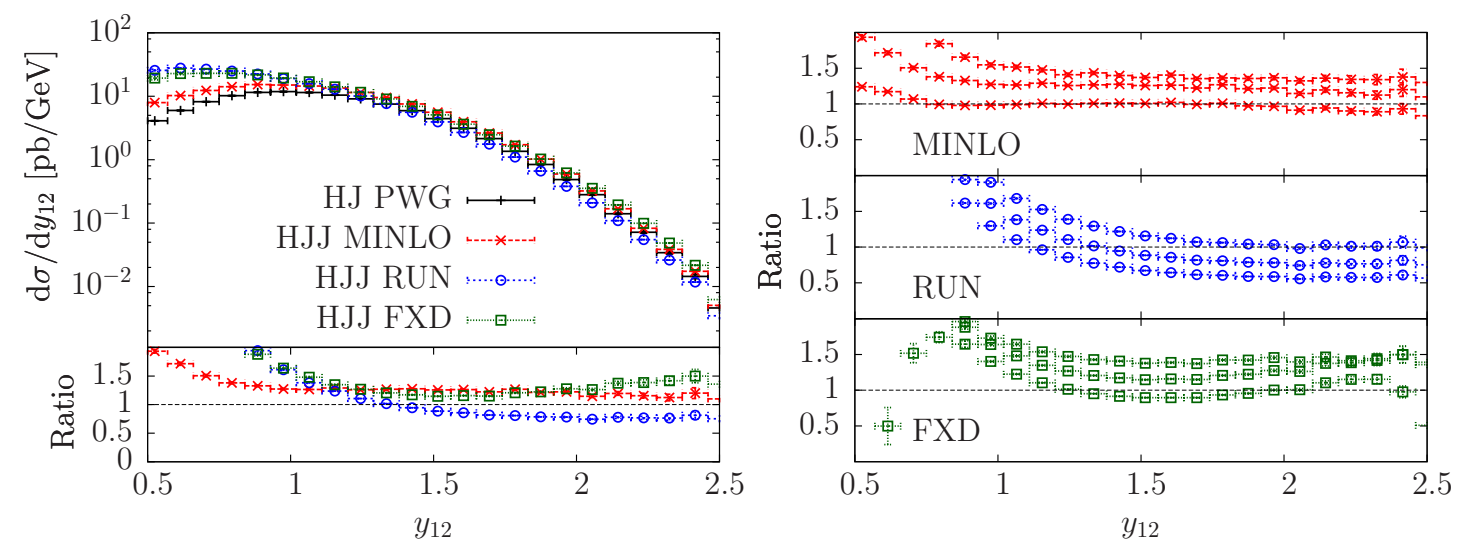

Figure 8. The differential jet rate $y_{12}$, defined as the value of the $k_{T}$ jet measure [14] for which events with two resolved jets are clustered into 1-jet events. Results are computed with the POWHEG BOX HJ generator (HJ PWG), the HJJ-MINLO result (HJJ MINLO), the HJJ with $\mu_{\mathrm{F}}=\mu_{\mathrm{R}}=\hat{H}_{T}$ (HJJ RUN), and HJJ with $\mu_{\mathrm{F}}=\mu_{\mathrm{R}}=M_{H}$ (HJJ FXD). To the right we show the ratio of each of the NLO HJJ results with respect to the NLO HJ POWHEG simulation, with the band either side of the central values indicating the combined renormalization and factorization scale uncertainty.
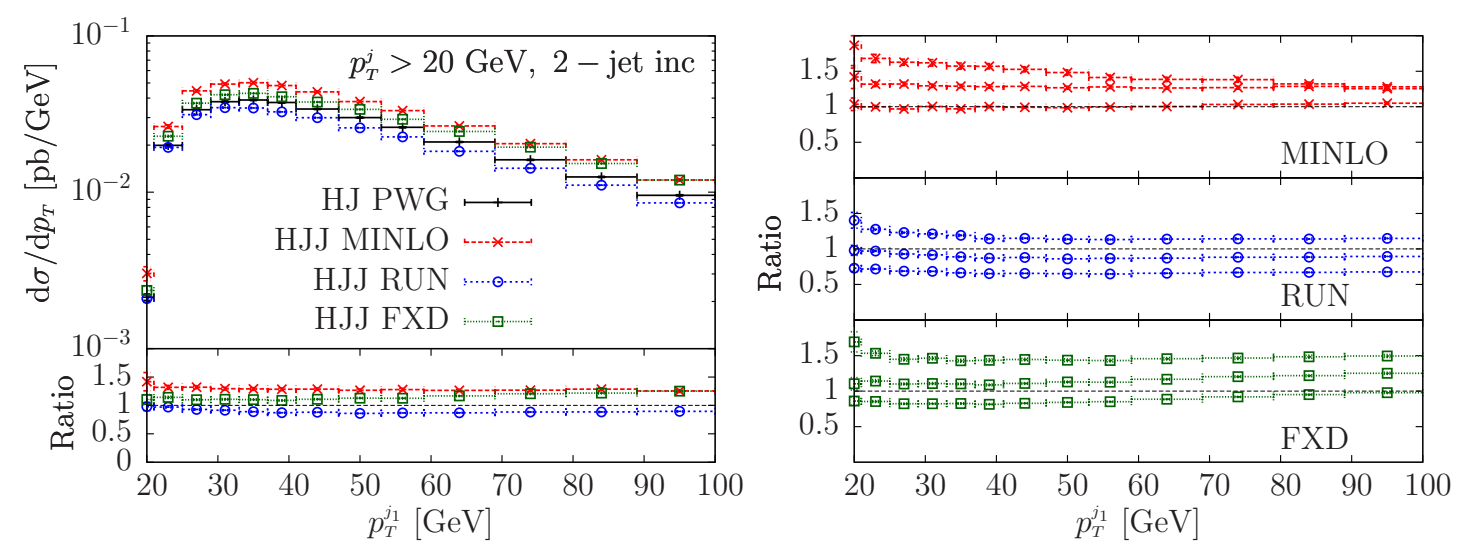

Figure 9. As in figure 8, for the transverse momentum of the leading jet in events with a Higgs boson and at least two jets with $p_{\mathrm{T}}>20 \mathrm{GeV}$.

MINLO result shrinks at high $p_{\mathrm{T}}$, while those of the NLO results using a more conventional scale choice do not. It is tempting to interpret this result as being due to the fact that the MINLO method yields smaller radiative corrections in the high $p_{\mathrm{T}}$ region, on account of its resummation of logarithms of the ratio of the widely different scales present in this observable - the jet $p_{\mathrm{T}}$ cut and $p_{\mathrm{T}}$ of the first jet. On the other hand, we must remember that the terms that are exponentiated in the Sudakov form factor are not subject to scale variation in our present procedure. Thus, we believe that much more practice with MINLO calculations is needed in order to substantiate this interpretation.

In figure 10 we show the transverse momentum distribution of the second jet. The $\hat{H}_{T}$ scale choice gives results below the MINLO ones, to an even larger extent than in the case of the leading jet. Again, choosing $\hat{H}_{T} / 2$ as central scale considerably improves the agreement, although not quite in a satisfactory way. Predictions with $\hat{H}_{T} / 2$ remain on the lower limit of the MINLO band for moderate transverse momenta. 

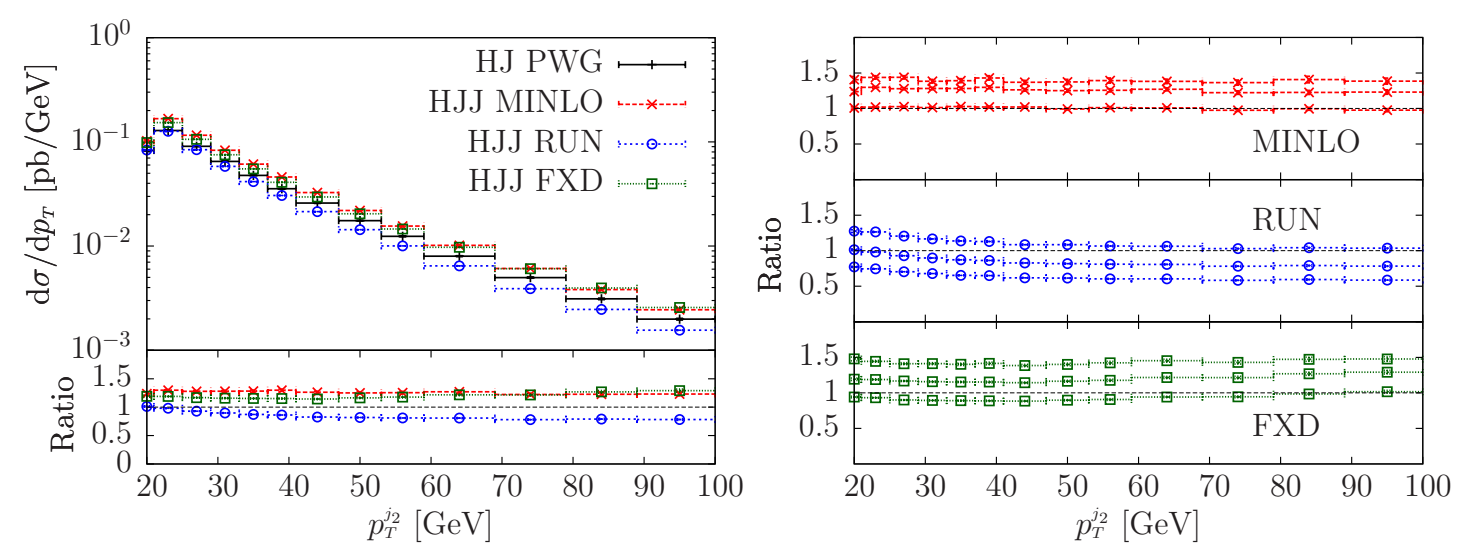

Figure 10. As in figure 8, for the transverse momentum of the next-to-leading jet in events with a Higgs boson and at least two jets with $p_{\mathrm{T}}>20 \mathrm{GeV}$.
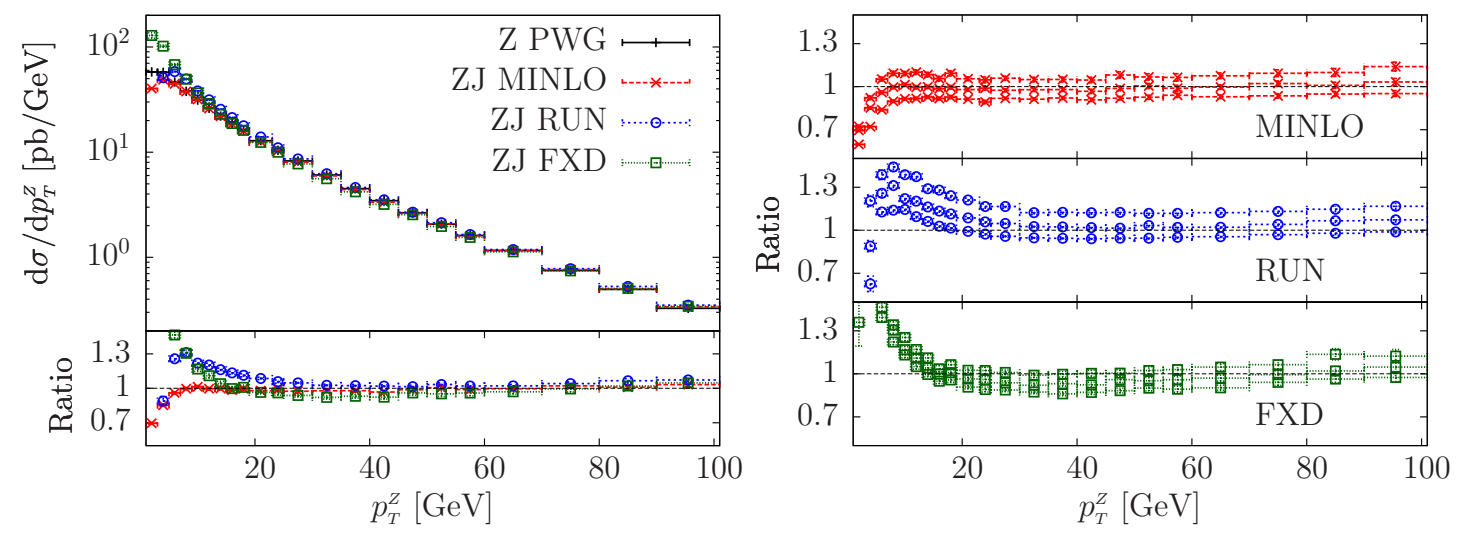

Figure 11. Transverse momentum spectrum of the $\mathrm{Z}$ boson computed with the POWHEG BOX $\mathrm{Z}$ generator, the ZJ-MINLO result, the ZJ default $\mu_{\mathrm{F}}=\mu_{\mathrm{R}}=p_{\mathrm{T}}^{Z}$ (ZJ RUN) and ZJ with $\mu_{\mathrm{F}}=$ $\mu_{\mathrm{R}}=M_{Z}$ (ZJ FXD). To the right we show the ratio of each of the NLO ZJ results with respect to the NLO Z POWHEG simulation, the band either side of the central values indicating the combined renormalization and factorization scale uncertainty.

\subsection{Z boson production}

\subsection{1 $Z$ boson production in association with one jet}

We begin by showing the transverse momentum of the $Z$ boson in figure 11, wherein we compare the predictions of the POWHEG $Z$ program interfaced to PYTHIA, MINLO ZJ and conventional NLO $Z+$ jet computations using two different scale choices: the mass of the $\mathrm{Z}$ boson and, separately, its transverse momentum. Scale uncertainty bands are presented in the accompanying plot to the right of the main distribution. As one can see, the MINLO result is closer to the POWHEG one for small transverse momenta. Observe, however, that now the agreement with POWHEG is not as good as in the Higgs case. It should be kept in mind, however, that the $Z$ Sudakov peak is located at much smaller values of the transverse momentum with respect to the Higgs case, in a region that is strongly influenced by cutoffs introduced to avoid the Landau pole in the perturbative calculation, and by shower cut-offs. 

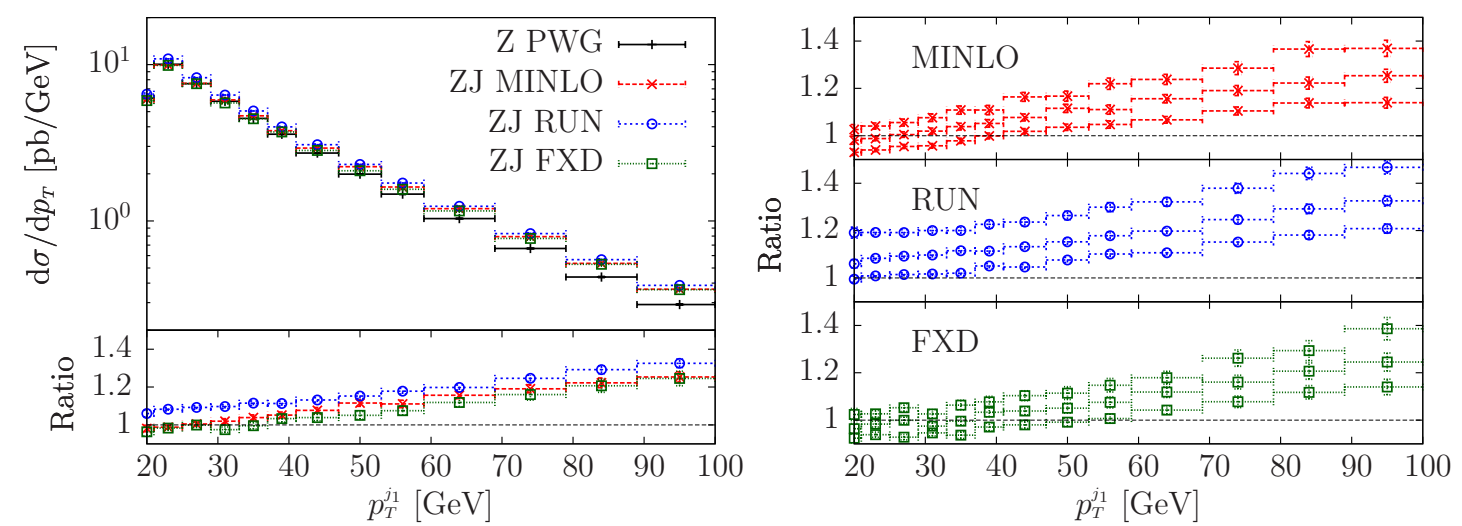

Figure 12. As figure 11 for the transverse momentum spectrum of the leading jet produced in $\mathrm{Z}$ boson production.
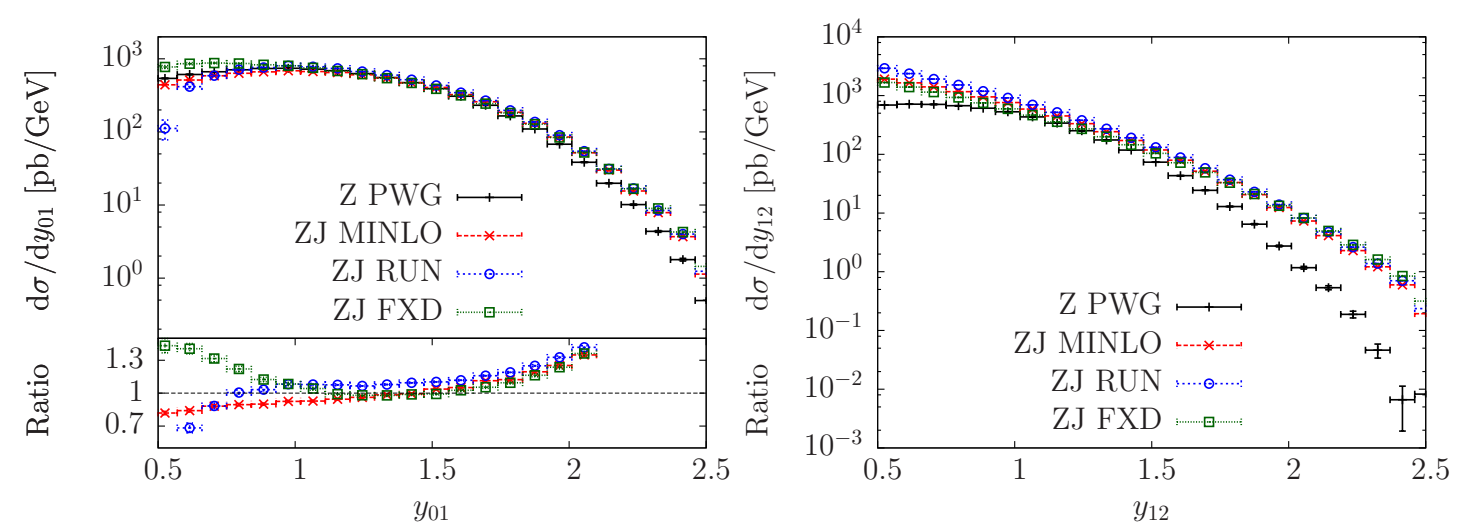

Figure 13. As figure 11 for the $0 \rightarrow 1$ and $1 \rightarrow 2$ differential jet rates in inclusive $\mathrm{Z}$ boson production.

In figure 12 we display the hardest jet transverse momentum. In this case, due to the $20 \mathrm{GeV}$ cut on the jet transverse momentum, we see that all prescriptions perform equally well.

In figure 13 we display the differential jet rates for the $0 \rightarrow 1$ and $1 \rightarrow 2$ transitions. In the first case, we see a more realistic behaviour of the MINLO result with respect to the conventional NLO predictions, while for the $1 \rightarrow 2$ transition, as noted previously in the case of Higgs production, all three methods are unreliable at small $y_{12}$. This behaviour is of course expected, since Sudakov resummation of the real radiation is absent in all but the POWHEG+PYTHIA prediction.

\subsection{2 $Z$ boson production in association with two jets}

In the case of $Z$ production in association with two jets, one does not expect a meaningful prediction for the $Z$ transverse momentum and for the $0 \rightarrow 1$ differential jet rate from regular NLO calculations with standard scale choices. As shown in figure 14 the MINLO result is instead sensible for both distributions.

Following our analysis for Higgs production, in figure 15 we have superimposed predictions from the MINLO ZJJ and conventional NLO $Z+2$ jets computations (with different 

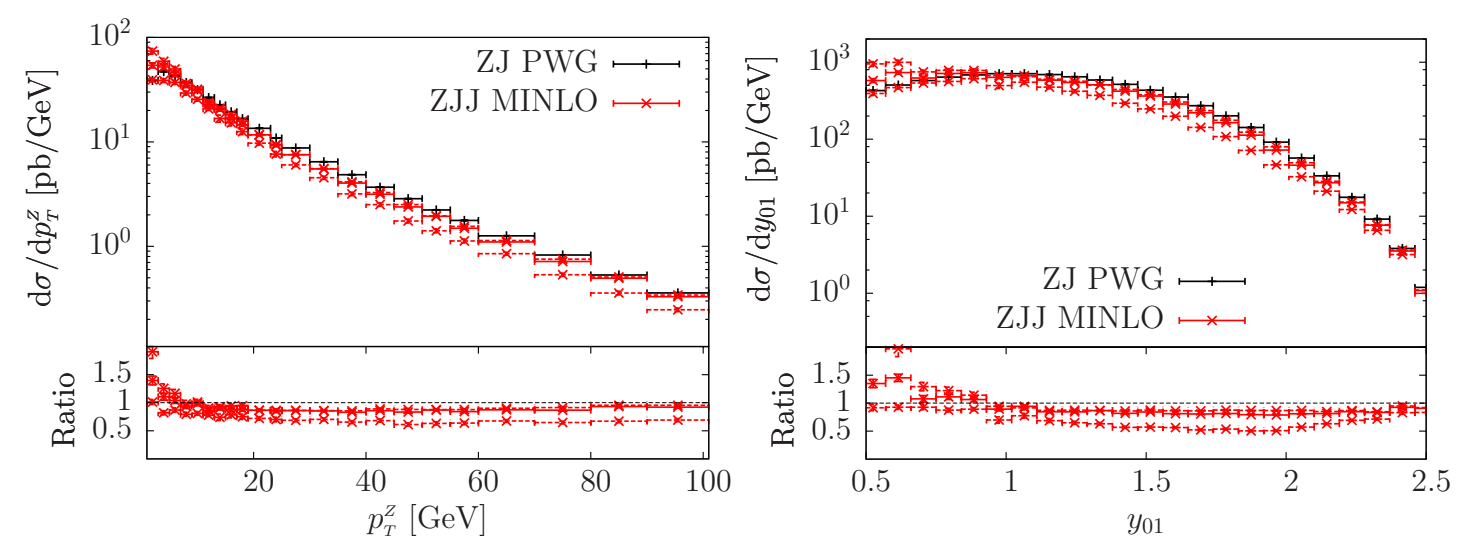

Figure 14. The transverse momentum of the $Z$ boson (left) and the differential jet rate $y_{01}$ (right), computed with the POWHEG BOX ZJ generator, augmented with the MINLO procedure and with the ZJJ-MINLO procedure.
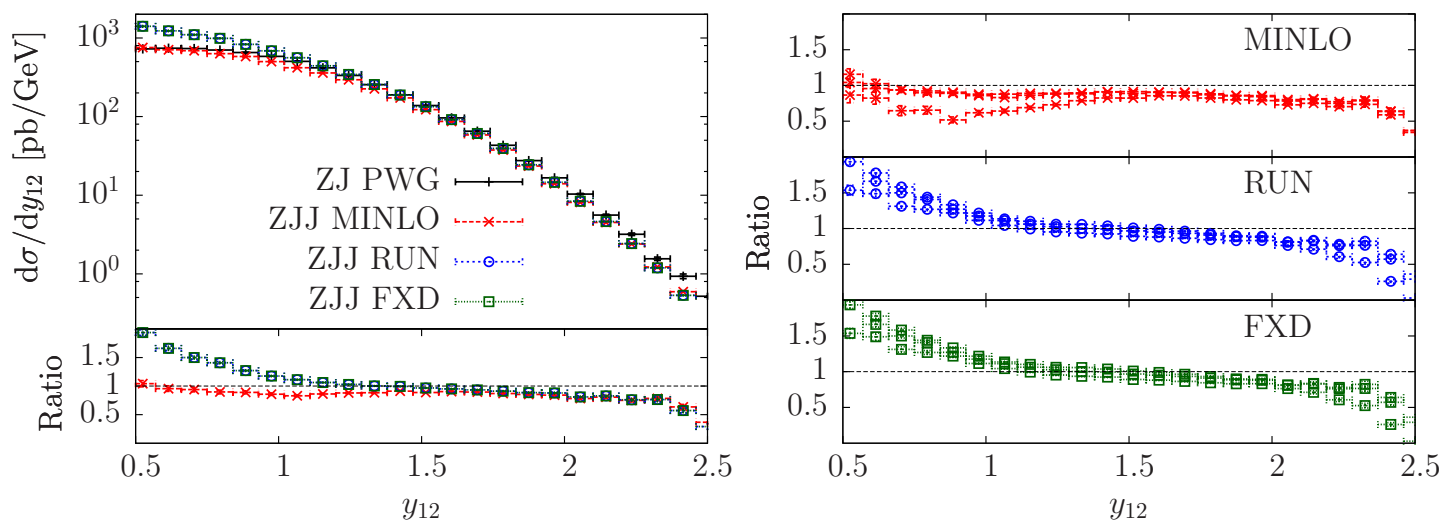

Figure 15. The differential jet rate $y_{12}$, computed with the ZJ POWHEG BOX simulation, augmented by the MINLO procedure (black), the MINLO ZJJ computation (red dashes), and conventional NLO ZJJ predictions with $\mu_{\mathrm{F}}=\mu_{\mathrm{R}}=\hat{H}_{T} / 2$ (blue dots) and $\mu_{\mathrm{F}}=\mu_{\mathrm{R}}=M_{Z}$ (fine green dots).

scale choices) on those of the ZJ POWHEG generator interfaced to PYTHIA, for the $1 \rightarrow 2$ differential jet rate. As in the case of Higgs production the Sudakov suppression effects built into the MINLO prediction are clearly manifest in the region $y_{12}<1$, where the regular NLO computations clearly depart from the fully resummed ZJ POWHEG result.

Finally, in figs 16 and 17 we show the transverse momentum distribution for the first and second jet, in events with at least two jets. In these figures we see that, when cuts are imposed to stay away from the Sudakov regions, all methods are in reasonable agreement.

\section{Conclusions}

In this paper we have formulated a method for the choice of scales and for the inclusion of Sudakov form factors in NLO calculations of processes involving jet production. The method proposed is such that the Born term is evaluated using the CKKW prescription, and the real and virtual corrections are added in such a way that formal NLO accuracy is maintained and the good features of the CKKW Born result are not spoiled. 

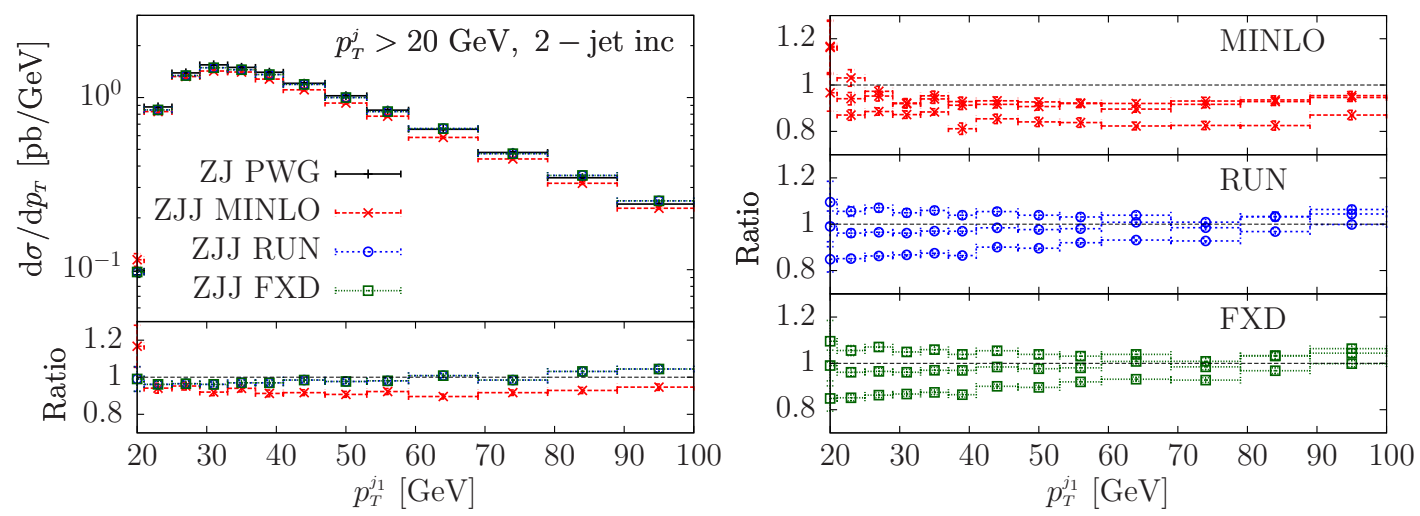

Figure 16. The transverse momentum of the leading jet in events comprising the $Z$ boson and at least two jets with $p_{\mathrm{T}}>20 \mathrm{GeV}$. As in figure 15 , on the left we compare the prediction of the ZJ POWHEG simulation, augmented by the MINLO procedure (black), to those of the MINLO ZJJ computation (red dashes), and conventional NLO ZJJ predictions with $\mu_{\mathrm{F}}=\mu_{\mathrm{R}}=\hat{H}_{T} / 2$ (blue dots) and $\mu_{\mathrm{F}}=\mu_{\mathrm{R}}=M_{Z}$ (fine green dots). To the right we show the ratio of each of the NLO ZJJ results with respect to the NLO ZJ POWHEG simulation, with the band either side of the central values indicating the combined renormalization and factorization scale uncertainty.
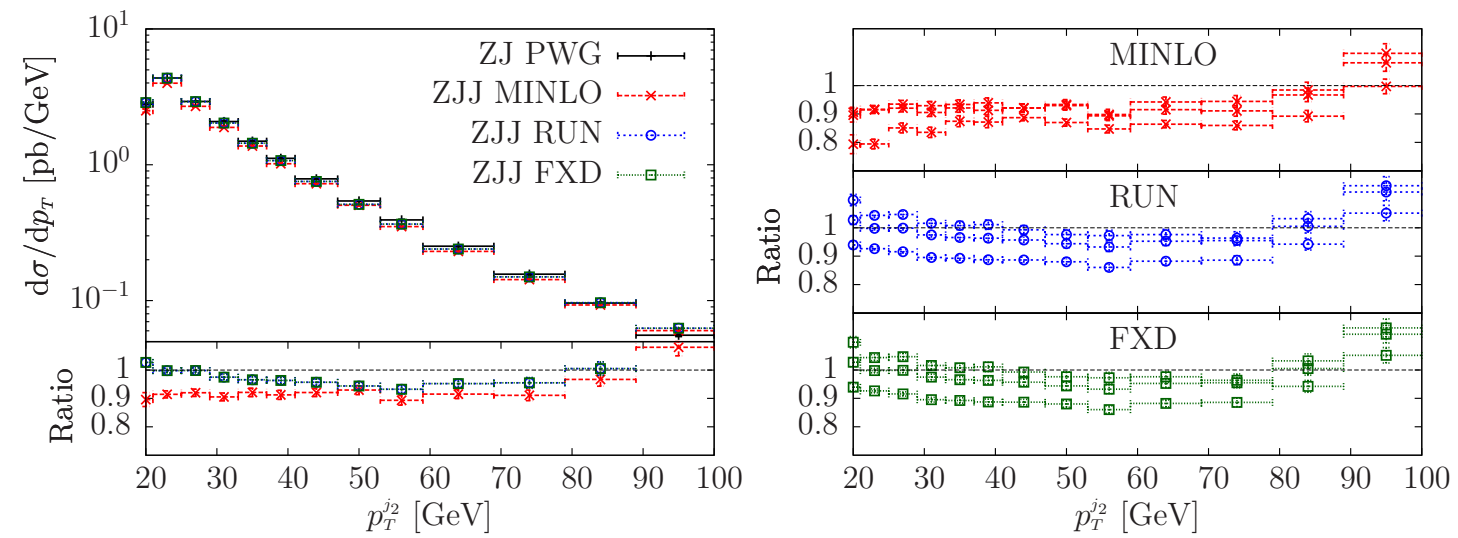

Figure 17. As in figure 16 for the transverse momentum of the next-to-leading jet in inclusive $Z$ boson production.

We have examined the performance of our method (MINLO for Multi-scale improved NLO) in Higgs and $Z$ boson production in association with up to two jets. We have observed the following properties of the MINLO method as compared to conventional NLO computations:

- The MINLO results are well behaved in the Sudakov regions for a large class of distributions, where instead the NLO results with standard choice of scale display large instabilities and breakdown. Although we do not expect the MINLO result to maintain NLO accuracy in the Sudakov regions, we clearly see the advantage that when these regions are approached, large Sudakov logarithms are exponentiated properly for a large class of observables.

- Away from the Sudakov regions, the MINLO method performs similarly to regular NLO computations using standard scale choices without Sudakov form factors. 
- In general, NLO results using popular prescriptions for the scale choice, like $\hat{H}_{T} / 2$, that tend to favour high scales, are in better agreement with the MINLO results. We attribute this behaviour as being due to the fact that larger scales in general lead to smaller cross sections, thus compensating for the lack of genuine Sudakov suppression.

The method we propose has the further advantage of great simplicity. All the formulae needed to implement it are given in the present work, and their POWHEG BOX implementation will soon be made publicly available.

The advantages of the MINLO method are strictly correlated with the advantages of the corresponding CKKW procedure. Thus, in the context of POWHEG simulations including parton showers, augmented with the MINLO procedure, we expect improved behaviour in Sudakov regions where more than one invariant becomes small, regardless of the observable under consideration. This parallels the fact that the CKKW method used in conjunction with a parton shower generator yields an improved description of the Sudakov effects for all observables. If the MINLO procedure is instead used in the context of a bare NLO calculation, we expect an improvement for observables that depend upon the final state pseudo-parton obtained using the $k_{\mathrm{T}}$ clustering algorithm, at the stage where we have a number of jets equal to the number $n$ of light partons accompanying the primary process at the Born level (e.g. $n=1$ for $H+1$ jet and $Z+1$ jet, and $n=2$ for $H+2$ jets and $Z+2$ jets).

Distributions that do not satisfy the above requirements are not well described by the MINLO procedure alone. However, we stress again that the MINLO procedure adopted in conjunction with a NLO+PS method like POWHEG or MC@NLO should yield an improved resummation of Sudakov logarithms for all observables.

We thus have been led in this study to conclude that finding an optimal scale in multiscale processes requires the inclusion of Sudakov form factors, whose subleading terms are required to compensate scale mismatch between nearby vertices. This leads formally to improved accuracy for a large class of distributions. In turn, the inclusion of the Sudakov form factor acts correctly for all observables only if we match the NLO calculation to a parton shower algorithm.

As a last point, we remark that many variations can be made on the method that we have proposed, that do not affect neither the NLO accuracy, nor the logarithmic resummation. One example is the scale chosen in the $(N+1)^{\text {th }}$ power of $\alpha_{\mathrm{S}}$ in the virtual, real and Sudakov subtraction term. The method that we propose is completely new, and in the present work we simply present it with a definite choice among all possible options. We will thus leave the exploration of all possible alternatives, and eventual refinements of our prescription, to future work.

\section{Acknowledgments}

We thank Gavin Salam and Bryan Webber for useful discussions. We are also grateful to Emanuele Re for providing virtual matrix elements squared for $\mathrm{Z}+$ dijet production at one phase space point. 
G.Z. is grateful to CERN and to the INFN in Milano-Bicocca for hospitality while part of this work was carried out. G.Z. is supported by the British Science and Technology Facilities Council. G.Z. and P.N. acknowledge the support also of grant PITN-GA-2010264564 from the European Commission.

\section{A NLL improved Sudakov}

We have used the following expression for the Sudakov exponent

$$
\log \Delta_{i}\left(Q_{0}, Q\right)=-\int_{Q_{0}}^{Q} \frac{\mathrm{d} q^{2}}{q^{2}}\left[\left(a\left(q^{2}\right) A_{1}^{(i)}+a^{2}\left(q^{2}\right) A_{2}^{(i)}\right) \log \frac{Q^{2}}{q^{2}}+a\left(q^{2}\right) B_{1}^{(i)}\right]
$$

where

$$
\begin{aligned}
A_{1}^{(q)} & =C_{\mathrm{F}}, \quad B_{1}^{(q)}=-\frac{3}{2} C_{\mathrm{F}}, \quad A_{1}^{(g)}=C_{\mathrm{A}}, \quad B_{1}^{(g)}=-2 \pi b_{0}, \\
A_{2}^{(g / q)} & =A_{1}^{(g / q)}\left[\left(\frac{67}{18}-\frac{\pi^{2}}{6}\right) C_{\mathrm{A}}-\frac{5}{9} n_{\mathrm{f}}\right],
\end{aligned}
$$

and

$$
2 \pi a\left(q^{2}\right)=\alpha_{\mathrm{S}}\left(q^{2}\right)=\frac{1}{b_{0} \log \left(q^{2} / \Lambda_{\overline{\mathrm{MS}}}^{2}\right)}\left[1-\frac{b_{1}}{b_{0}^{2}} \frac{\log \log \left(q^{2} / \Lambda_{\overline{\mathrm{MS}}}^{2}\right)}{\log \left(q^{2} / \Lambda_{\overline{\mathrm{MS}}}^{2}\right)}\right]
$$

with

$$
b_{0}=\frac{33-2 n_{\mathrm{f}}}{12 \pi}, \quad b_{1}=\frac{153-19 n_{\mathrm{f}}}{24 \pi^{2}} .
$$

Equation (A.1) was integrated analytically and the result checked numerically. We have always used $n_{\mathrm{f}}=5$, and the five flavours expression of $\Lambda_{\overline{\mathrm{MS}}}$.

Open Access. This article is distributed under the terms of the Creative Commons Attribution License which permits any use, distribution and reproduction in any medium, provided the original author(s) and source are credited.

\section{References}

[1] P.M. Stevenson, Optimized perturbation theory, Phys. Rev. D 23 (1981) 2916 [InSPIRE].

[2] S.J. Brodsky, G.P. Lepage and P.B. Mackenzie, On the elimination of scale ambiguities in perturbative quantum chromodynamics, Phys. Rev. D 28 (1983) 228 [INSPIRE].

[3] S.J. Brodsky and L. Di Giustino, Setting the renormalization scale in QCD: the principle of maximum conformality, arXiv:1107.0338 [INSPIRE].

[4] S. Catani, F. Krauss, R. Kuhn and B. Webber, QCD matrix elements + parton showers, JHEP 11 (2001) 063 [hep-ph/0109231] [INSPIRE].

[5] M.L. Mangano, M. Moretti and R. Pittau, Multijet matrix elements and shower evolution in hadronic collisions: $W b \bar{b}+n$ jets as a case study, Nucl. Phys. B 632 (2002) 343 [hep-ph/0108069] [INSPIRE].

[6] L. Lönnblad, Correcting the color dipole cascade model with fixed order matrix elements, JHEP 05 (2002) 046 [hep-ph/0112284] [INSPIRE]. 
[7] F. Krauss, Matrix elements and parton showers in hadronic interactions, JHEP 08 (2002) 015 [hep-ph/0205283] [INSPIRE].

[8] S. Mrenna and P. Richardson, Matching matrix elements and parton showers with HERWIG and PYTHIA, JHEP 05 (2004) 040 [hep-ph/0312274] [INSPIRE].

[9] M. Mangano, Merging multijet matrix elements and shower evolution in hadronic collisions, http://mlm.web.cern.ch/mlm/talks/lund-alpgen.pdf (2004).

[10] S. Frixione and B.R. Webber, Matching NLO QCD computations and parton shower simulations, JHEP 06 (2002) 029 [hep-ph/0204244] [INSPIRE].

[11] P. Nason, A new method for combining NLO QCD with shower Monte Carlo algorithms, JHEP 11 (2004) 040 [hep-ph/0409146] [INSPIRE].

[12] S. Frixione, P. Nason and C. Oleari, Matching NLO QCD computations with parton shower simulations: the POWHEG method, JHEP 11 (2007) 070 [arXiv: 0709.2092] [INSPIRE].

[13] S.D. Ellis and D.E. Soper, Successive combination jet algorithm for hadron collisions, Phys. Rev. D 48 (1993) 3160 [hep-ph/9305266] [INSPIRE].

[14] S. Catani, Y.L. Dokshitzer, M. Seymour and B. Webber, Longitudinally invariant $K_{t}$ clustering algorithms for hadron hadron collisions, Nucl. Phys. B 406 (1993) 187 [INSPIRE].

[15] M. Rubin, G.P. Salam and S. Sapeta, Giant QCD K-factors beyond NLO, JHEP 09 (2010) 084 [arXiv: 1006.2144] [INSPIRE].

[16] S. Alioli, P. Nason, C. Oleari and E. Re, A general framework for implementing NLO calculations in shower Monte Carlo programs: the POWHEG BOX, JHEP 06 (2010) 043 [arXiv: 1002.2581] [INSPIRE].

[17] D. de Florian, M. Grazzini and Z. Kunszt, Higgs production with large transverse momentum in hadronic collisions at next-to-leading order, Phys. Rev. Lett. 82 (1999) 5209 [hep-ph/9902483] [INSPIRE].

[18] V. Ravindran, J. Smith and W. Van Neerven, Next-to-leading order QCD corrections to differential distributions of Higgs boson production in hadron hadron collisions, Nucl. Phys. B 634 (2002) 247 [hep-ph/0201114] [INSPIRE].

[19] J.M. Campbell, R.K. Ellis and G. Zanderighi, Next-to-leading order Higgs + 2 jet production via gluon fusion, JHEP 10 (2006) 028 [hep-ph/0608194] [INSPIRE].

[20] J.M. Campbell, R.K. Ellis and C. Williams, Hadronic production of a Higgs boson and two jets at next-to-leading order, Phys. Rev. D 81 (2010) 074023 [arXiv:1001.4495] [INSPIRE].

[21] J.M. Campbell et al., NLO Higgs boson production plus one and two jets using the POWHEG BOX, MadGraph 4 and MCFM, JHEP 07 (2012) 092 [arXiv: 1202.5475] [INSPIRE].

[22] S. Alioli, P. Nason, C. Oleari and E. Re, Vector boson plus one jet production in POWHEG, JHEP 01 (2011) 095 [arXiv: 1009.5594] [INSPIRE].

[23] E. Re, NLO corrections merged with parton showers for $Z+2$ jets production using the POWHEG method, JHEP 10 (2012) 031 [arXiv:1204.5433] [INSPIRE].

[24] W. Giele, E.N. Glover and D.A. Kosower, Higher order corrections to jet cross-sections in hadron colliders, Nucl. Phys. B 403 (1993) 633 [hep-ph/9302225] [INSPIRE].

[25] Z. Bern, L.J. Dixon and D.A. Kosower, One loop amplitudes for $e^{+} e^{-}$to four partons, Nucl. Phys. B 513 (1998) 3 [hep-ph/9708239] [InSPIRE]. 
[26] J.M. Campbell and R.K. Ellis, Next-to-leading order corrections to $W+2$ jet and $Z+2$ jet production at hadron colliders, Phys. Rev. D 65 (2002) 113007 [hep-ph/0202176] [INSPIRE].

[27] J. Pumplin et al., New generation of parton distributions with uncertainties from global QCD analysis, JHEP 07 (2002) 012 [hep-ph/0201195] [INSPIRE].

[28] A. Martin, W. Stirling, R. Thorne and G. Watt, Parton distributions for the LHC, Eur. Phys. J. C 63 (2009) 189 [arXiv:0901.0002] [InSPIRE].

[29] M. Cacciari, G.P. Salam and G. Soyez, FastJet user manual, Eur. Phys. J. C 72 (2012) 1896 [arXiv: 1111.6097] [INSPIRE].

[30] T. Sjöstrand, S. Mrenna and P.Z. Skands, PYTHIA 6.4 physics and manual, JHEP 05 (2006) 026 [hep-ph/0603175] [INSPIRE].

[31] C. Berger et al., Precise predictions for $W+4$ jet production at the Large Hadron Collider, Phys. Rev. Lett. 106 (2011) 092001 [arXiv: 1009.2338] [INSPIRE].

[32] G.P. Salam, private communication.

[33] S. Dittmaier et al., Handbook of LHC Higgs cross sections: 2. Differential distributions, arXiv:1201.3084 [INSPIRE].

[34] G. Bozzi, S. Catani, G. Ferrera, D. de Florian and M. Grazzini, Transverse-momentum resummation: A Perturbative study of $Z$ production at the Tevatron, Nucl. Phys. B 815 (2009) 174 [arXiv:0812.2862] [INSPIRE]. 